\title{
ILCEA
}

Revue de l'Institut des langues et cultures

d'Europe, Amérique, Afrique, Asie et Australie

$29 \mid 2017$

Les femmes en Russie : parcours, mythes et représentations

\section{АВДОТЬЯ ПАНАЕВА МЕЖДУ ПУБЛИЧНЫМ И ЛИЧНЫМ ПРОСТРАНСТВОМ}

Avdotia Panaeva entre l'espace public et l'espace intime [par Olga Kafanova]

\section{Ольга Кафанова}

\section{OpenEdition}

Journals

Édition électronique

URL : http://journals.openedition.org/ilcea/4296

DOI : 10.4000/ilcea.4296

ISSN : 2101-0609

\section{Éditeur}

UGA Éditions/Université Grenoble Alpes

\section{Édition imprimée}

ISBN : 978-2-37747-007-5

ISSN : 1639-6073

\section{Référence électronique}

Ольга Кафанова, «Авдотья Панаева между публичным и личным пространством », ILCEA [Онлайн], 29 | 2017, Выложить онлайн 30 јuin 2017, Наводить справки в 20 avril 2019. URL : http:// journals.openedition.org/ilcea/4296; DOI : 10.4000/ilcea.4296

Ce document a été généré automatiquement le 20 avril 2019

(C) ILCEA 


\title{
АВДОТЬЯ ПАНАЕВА МЕЖДУ ПУБЛИЧНЫМ И ЛИЧНЫМ ПРОСТРАНСТВОМ
}

Avdotia Panaeva entre l'espace public et l'espace intime [par Olga Kafanova]

\author{
Ольга Кафанова
}

\section{Введение}

СУДЬБА АВДОТЬИ ЯКОВЛЕВНЫ ПАНАЕВОЙ (1821-1893) - ЛИЧНАЯ И ТВОРЧЕСКАЯ - ОЧЕНЬ ПОКАЗАТЕЛЬНА ДЛЯ ХАРАКТЕРИСТИКИ ЧАСТНОГО И ПУБЛИЧНОГО СУЩЕСТВОВАНИЯ РУССКОЙ ЖЕНЩИНЫ-ЛИТЕРАТОРА В РОССИИ ВТОРОЙ ПОЛОВИНЫ ХІХ В. ХУДОЖЕСТВЕННОЕ НАСЛЕДИЕ А.ПАНАЕВОЙ ОТРАЖАЕТ СПЕЦИФИКУ ЖЕНСКОГО МИРОВИДЕНИЯ, СТАНОВЛЕНИЕ КОТОРОГО ОСУЩЕСТВЛЯЛОСЬ В ДИАЛОГЕ С ИДЕЙНОФИЛОСОФСКИМ И ЛИТЕРАТУРНЫМ ДИСКУРСОМ ЕЕ ВРЕМЕНИ. КАТЕГОРИЯ ГЕНДЕРА - ОБЪЕКТИВНАЯ СОСТАВЛЯЮЩАЯ СОЦИОКУЛЬТУРНОГО БЫТИЯ ЧЕЛОВЕКА - ПОЗВОЛЯЕТ ПО-НОВОМУ ОСМЫСЛИТЬ ЛОГИКУ РАЗВИТИЯ ЛИТЕРАТУРНОГО ПРОЦЕССА, МЕХАНИЗМЫ СОЗДАНИЯ И ВОСПРИЯТИЯ ХУДОЖЕСТВЕННОГО ТЕКСТА.

2 ТВОРЧЕСТВО А. ПАНАЕВОЙ, КАК И ДРУГИХ ЖЕНЩИН-ПИСАТЕЛЬНИЦ ХІХ ВЕКА, ПРИЧИСЛЯЮТ К ЛИТЕРАТУРЕ «ВТОРОГО РЯДА», БЕЗ ИЗУЧЕНИЯ КОТОРОЙ НЕВОЗМОЖНО ПОНЯТЬ СВОЕОБРАЗИЕ ЛИТЕРАТУРНОГО ПРОЦЕССА. С ОДНОЙ СТОРОНЫ, В ЖЕНСКОЙ ПРОЗЕ НАИБОЛЕЕ РЕЛЬЕФНО ОТРАЖАЮТСЯ ОСНОВНЫЕ ТЕНДЕНЦИИ РАЗВИТИЯ ПРЕОБЛАДАЮЩЕЙ ЭСТЕТИЧЕСКОЙ ПАРАДИГМЫ, А С ДРУГОЙ - ВЫРАБАТЫВАЮТСЯ НОВЫЕ ХУДОЖЕСТВЕННЫЕ ПРИНЦИПЫ, КОТОРЫЕ В ПРОИЗВЕДЕНИЯХ «БОЛЬШИХ» ХУДОЖНИКОВ ДОСТИГАЮТ УЖЕ СОВЕРШЕННОГО УРОВНЯ.

ПРОИЗВЕДЕНИЯ А. ПАНАЕВОЙ (БОЛЕЕ ДВАДЦАТИ ПОВЕСТЕЙ И РОМАНОВ, А ТАКЖЕ СОЗДАННЫЕ ЕЮ В КОНЦЕ ЖИЗНИ «ВОСПОМИНАНИЯ») ПУБЛИКОВАЛИСЬ В КРУПНЕЙШИХ ПЕРИОДИЧЕСКИХ ИЗДАНИЯХ ПЕТЕРБУРГА («СОВРЕМЕННИК», «НИВА», «ЖИВОПИСНОЕ 
ОБОЗРЕНИЕ», «ИСТОРИЧЕСКИЙ ВЕСТНИК») И ПОЛЬЗОВАЛИСЬ ПОПУЛЯРНОСТЬЮ У ЧИТАТЕЛЕЙ. ОДНАКО ОЦЕНКА ТВОРЧЕСТВА А. ПАНАЕВОЙ В КРИТИКЕ ХІХ ВЕКА ХАРАКТЕРИЗУЕТСЯ НЕОДНОЗНАЧНОСТЬЮ: ОТ ПРИЗНАНИЯ ЕГО ОТДЕЛЬНЫХ ХУДОЖЕСТВЕННЫХ ДОСТОИНСТВ (В.БЕЛИНСКИЙ, А.ГРИГОРЬЕВ ОТМЕЧАЛИ В ЕЕ ПРОИЗВЕДЕНИЯХ НОВИЗНУ СОДЕРЖАНИЯ» И ЖЕНСКИХ ОБРАЗОВ) ДО ПОЛНОГО НЕПРИЯТИЯ (Д. ПИСАРЕВ). ФОРМИРОВАНИЕ ИДЕЙНО-ЭСТЕТИЧЕСКОЙ ПОЗИЦИИ А. ПАНАЕВОЙ НЕОТДЕЛИМО ОТ ФИЛОСОФСКО-ИДЕОЛОГИЧЕСКОГО ДИСКУРСА $1840-1860-\mathrm{X}$ ГОДОВ, В КОТОРОМ РАССМАТРИВАЛИСЬ ОСНОВНЫЕ КОНЦЕПЦИИ ОТНОШЕНИЙ ПОЛОВ И ОРГАНИЗАЦИИ СЕМЬИ - ТРАДИЦИОННЫЕ (ХРИСТИАНСКАЯ, ПАТРИАРХАТНАЯ) И ИННОВАЦИОННЫЕ. ПРЕДСТАВЛЕНИЯ А. ПАНАЕВОЙ О ПРИРОДЕ ЛЮБВИ И НРАВСТВЕННОЙ СТОРОНЕ СУПРУЖЕСКОГО СОЮЗА, ОПРЕДЕЛИВШИЕ ЕЕ СОБСТВЕННУЮ ЛИЧНУЮ ЖИЗНЬ, А ТАКЖЕ СЮЖЕТНО-МОТИВНУЮ ОРГАНИЗАЦИЮ ЕЕ ПРОЗЫ, ФОРМИРОВАЛИСЬ ПОД ВОЗДЕЙСТВИЕМ ФИЛОСОФИИ ФРАНЦУЗСКОГО УТОПИЧЕСКОГО И ХРИСТИАНСКОГО СОЦИАЛИЗМА.

\section{Дискуссия 1840-х гг. о любовно-семейной этике}

«СОРОКОВЫЕ ГОДЫ» ХІХ ВЕКА, ВРЕМЯ СТАНОВЛЕНИЯ А. ПАНАЕВОЙ КАК ЛИЧНОСТИ И ХУДОЖНИКА, ЯВЛЯЕТСЯ НЕ ТОЛЬКО ЭПОХОЙ РАДИКАЛИЗМА В РУССКОЙ КУЛЬТУРЕ, НО И ОСОБО ЗНАЧИМЫМ ПЕРИОДОМ ЛОМКИ НРАВСТВЕННЫХ СТЕРЕОТИПОВ, РАЗРУШЕНИЯ ТРАДИЦИОННЫХ МОДЕЛЕЙ ПОВЕДЕНИЯ МУЖЧИНЫ И ЖЕНЩИНЫ В БРАКЕ, ПЕРЕСМОТРА КОНЦЕПЦИИ «ПАДШЕЙ» ЖЕНЩИНЫ, ОТКАЗА ОТ ИДЕИ НЕЗЫБЛЕМОСТИ СУПРУЖЕСКОГО СОЮ3А.

В ПРЕДЫДУЩИЙ ИСТОРИКО-КУЛЬТУРНЫЙ ПЕРИОД - 1830-Е ГГ. - ИДЕАЛ РУССКОЙ ЖЕНЩИНЫ ВЫРАЖАЛА ТАТЬЯНА ЛАРИНА, ГЕРОИНЯ «ЕВГЕНИЯ ОНЕГИНА» А. С. ПУШКИНА. ЭТОТ ОБРАЗ РЕПРЕЗЕНТИРОВАЛ НЕСАМОСТОЯТЕЛЬНОСТЬ РУССКОЙ ДЕВУШКИ В ВЫБОРЕ МУЖА, ВЕРНОСТЬ ЗАМУЖНЕЙ ЖЕНЩИНЫ ОБЕТУ, ПРОИЗНЕСЕННОМУ ПЕРЕД АЛТАРЕМ И, СЛЕДОВАТЕЛЬНО, НЕРАСТОРЖИМОСТЬ БРАКА: «НО Я ДРУГОМУ ОТДАНА И БУДУ ВЕК ЕМУ ВЕРНА.» ЛЮБОВЬ, ВЗАИМНОЕ ВЛЕЧЕНИЕ НЕ ПРИНИМАЛИСЬ ВО ВНИМАНИЕ, А ГЛАВНОЕ ПРЕДНАЗНАЧЕНИЕ БРАКА ВИДЕЛОСЬ В ПРОДОЛЖЕНИИ РОДА. МОДЕЛЬ ПОВЕДЕНИЯ ПУШКИНСКОЙ ГЕРОИНИ ПРЕЖДЕ ВСЕГО ПРИВЕТСТВОВАЛИ МУЖЧИНЫ, В ТО ВРЕМЯ КАК ЖЕНЩИНЫ НАЧАЛИ ВСЕ БОЛЕЕ ЯВНО ВЫРАЖАТЬ СВОЮ ОППОЗИЦИЮ, КОТОРАЯ ПРОЯВЛЯЛАСЬ В НОВЫХ СЮЖЕТАХ И НРАВСТВЕННОМ ПАФОСЕ ИХ ПРОИЗВЕДЕНИЙ (ПОВЕСТИ Е. А. ГАН «ИДЕАЛ», «СУД СВЕТА») ИЛИ ДАЖЕ ПОВЕДЕНИИ (ПРИМЕРОМ МОЖЕТ СЛУЖИТЬ А. П. КЕРН, ПОКИНУВШАЯ СВОЕГО НЕЛЮБИМОГО МУЖА, ГЕНЕРАЛА Е. Ф. КЕРНА, ОБРАТИВШЕГОСЯ ЗА ПОМОЩЬЮ В ЭТОМ ДЕЛИКАТНОМ ДЕЛЕ К ИМПЕРАТОРУ НИКОЛАЮ І).

В 1840-Е ГГ. РУССКИЕ ЗАПАДНИКИ ВО ГЛАВЕ С ВИССАРИОНОМ БЕЛИНСКИМ УСВАИВАЮТ И С ВООДУШЕВЛЕНИЕМ ПРОПОВЕДУЮТ НОВЫЕ НРАВСТВЕННЫЕ ИДЕИ О ЛЮБВИ И БРАКЕ, ВЗАИМООТНОШЕНИЯХ МУЖЧИНЫ И ЖЕНЩИНЫ В ЛЮБОВНОМ БЫТУ, ПРИНЕСЕННЫЕ В РОССИЮ С ПРОИЗВЕДЕНИЯМИ ЖОРЖ САНД. НАЧАЛАСЬ ДИСКУССИЯ, НЕ ОСТАВИВШАЯ РАВНОДУШНЫМ НИ ОДНОГО КУЛЬТУРНОГО ЧЕЛОВЕКА В РОССИИ. БЕЛИНСКИЙ, КРИТИК СТРАСТНЫЙ, УВЛЕКАЮЩИЙСЯ И ТОНКО ЧУВСТВУЮЩИЙ НОВЫЕ НРАВСТВЕННЫЕ ВЕЯНИЯ, ОБЛЕЧЕННЫЕ В ЯРКОЕ ХУДОЖЕСТВЕННОЕ СЛОВО, УВИДЕЛ В САНД ПРОВОЗВЕСТНИЦУ КАКОЙ-ТО НОВОЙ ПРАВДЫ, ЧУТЬ ЛИ НЕ ПОСЛЕДНЕЙ ИСТИНЫ О ЧЕЛОВЕКЕ И ЕГО МЕСТЕ В МИРЕ И ОБЩЕСТВЕ. 
К ОТКРЫТИЯМ, ПОЧЕРПНУТЫМ ИЗ ИДЕЙ ЖОРЖ САНД, ОТНОСИЛОСЬ УБЕЖДЕНИЕ БЕЛИНСКОГО В ТОМ, ЧТО ЛЮБОВНО-СЕМЕЙНАЯ СФЕРА ЯВЛЯЕТСЯ ОДНОЙ ИЗ ВАЖНЕЙШИХ ОСНОВ ОБЩЕСТВЕННОЙ ЖИЗНИ И ЧЕЛОВЕЧЕСКОГО БЫТИЯ. ЭТИМ ОБЪЯСНЯЕТСЯ ТАКОЕ НЕОБЫЧАЙНО ОБОСТРЕННОЕ ВНИМАНИЕ ЛЮДЕЙ «СОРОКОВЫХ ГОДОВ» К ПРОБЛЕМАМ ЛЮБВИ И БРАКА (КАФАНОВА, 1998b: 78-87). С ОДНОЙ СТОРОНЫ, ИСТИННАЯ ЛЮБОВЬ ПРИЗНАВАЛАСЬ ЕДВА ЛИ НЕ ЕДИНСТВЕННЫМ КРИТЕРИЕМ НРАВСТВЕННОГО ПОВЕДЕНИЯ ЧЕЛОВЕКА В ЛЮБОВНО-СЕМЕЙНОЙ КОЛЛИЗИИ. ОТСЮДА ВЫТЕКАЛИ НОВЫЕ ЭТИЧЕСКИЕ ОЦЕНКИ СУПРУЖЕСКОЙ ИЗМЕНЫ. ПОСТУПОК ЖЕНЩИНЫ, КОТОРАЯ, ПОДОБНО ТАТЬЯНЕ ЛАРИНОЙ, ПРЕДПОЧИТАЛА НЕЛЮБИМОГО МУЖА ЛЮБИМОМУ МУЖЧИНЕ ВО ИМЯ ИСПОЛНЕНИЯ ДОЛГА, ВОСПРИНИМАЛАСЬ ВИССАРИОНОМ БЕЛИНСКИМ, ПАВЛОМ АННЕНКОВЫМ, АЛЕКСАНДРОМ ГЕРЦЕНОМ, НИКОЛАЕМ ОГАРЕВЫМ, ИВАНОМ ПАНАЕВЫМ И ДР. КАК ЛИЦЕМЕРИЕ И ХАНЖЕСТВО (ИЛИ ДАЖЕ КАК «УЗАКОНЕННАЯ ПРОСТИТУЦИЯ» СОГЛАСНО МАКСИМАЛИСТСКОЙ КОНЦЕПЦИИ ВАСИЛИЯ БОТКИНА, ДРУГА БЕЛИНСКОГО).

С ДРУГОЙ СТОРОНЫ, ЭТИКА САМОПОЖЕРТВОВАНИЯ ПРЕДПИСЫВАЛАСЬ МУЖЧИНЕ, КОТОРЫЙ КАК БЫ ДОЛЖЕН БЫЛ ВЗЯТЬ НА СЕБЯ ОТВЕТСТВЕННОСТЬ ЗА НЕСОВЕРШЕННЫЕ ЗАКОНЫ, ОСОБЕННО УГНЕТАЮЩИЕ И ПРИНИЖАЮЩИЕ ЖЕНЩИНУ. РАСТОРЖЕНИЕ ЦЕРКОВНОГО БРАКА БЫЛО ПРОЦЕДУРОЙ КРАЙНЕ СЛОЖНОЙ, ПОЭТОМУ В СЛУЧАЕ ВОЗНИКНОВЕНИЯ ЛЮБОВНОГО ТРЕУГОЛЬНИКА БЛАГОРОДНЫЙ МУЖ РАДИ СЧАСТЬЯ ЛЮБИМОЙ ИМ ЖЕНЩИНЫ ДОЛЖЕН БЫЛ САМОУСТРАНИТЬСЯ. ЖОРЖ САНД С НОВЫХ НРАВСТВЕННЫХ ПОЗИЦИЙ РАССМОТРЕЛА КОЛЛИЗИЮ ПРЕЛЮБОДЕЯНИЯ И ВВЕЛА МОТИВ САМОУСТРАНЕНИЯ МУЖА В РОМАНЕ Jacques (1834). В СИТУАЦИИ ОБЩЕСТВЕННОЦЕРКОВНОГО УЩЕМЛЕНИЯ ЖЕНЩИНЫ В ПРАВАХ БЛАГОРОДНЫЙ МУЖЧИНА ОБЯЗАН ВЗЯТЬ НА СЕБЯ ЕЕ ЗАЩИТУ. А ТАК КАК ПРИРОДА ЛЮБВИ НЕПРЕДСКАЗУЕМА И НЕ ПОДДАЕТСЯ РАЦИОНАЛЬНОМУ ОСМЫСЛЕНИЮ, ТО НЕЛЬЗЯ КАРАТЬ ЗА ИЗМЕНУ. ПОСКОЛЬКУ ПОРЯДОЧНЫЙ МУЖЧИНА В ЛЮБВИ ДОЛЖЕН БЫТЬ, ПРЕЖДЕ ВСЕГО, ДРУГОМ СВОЕЙ ЖЕНЕ И ДУМАТЬ О ЕЕ БЛАГЕ, ТО ЕДИНСТВЕННЫМ ВЫХОДОМ ИЗ ЛЮБОВНОГО ТРЕУГОЛЬНИКА СТАНОВИТСЯ ЕГО ДОБРОВОЛЬНОЕ САМОУСТРАНЕНИЕ. ЭТОТ МОТИВ ПОЛУЧИЛ РАЗВИТИЕ В РУССКОЙ ЛИТЕРАТУРЕ В ТАКИХ ПРОИЗВЕДЕНИЯХ, КАК «ПОЛИНЬКА САКС» А.В. ДРУЖИНИНА, «ПОДВОДНЫЙ КАМЕНЬ» М. В. АВДЕЕВА, «ЧТО ДЕЛАТЬ?» Н.Г. ЧЕРНЫШЕВСКОГО, «ЖИВОЙ ТРУП» Л. Н. ТОЛСТОГО И ДР. ВЛИЯНИЕ ЖОРЖ САНД ДАЛЕКО ВЫХОДИЛО ЗА ПРЕДЕЛЫ ЛИТЕРАТУРЫ, НО САМЫМ НЕПОСРЕДСТВЕННЫМ ОБРАЗОМ «АПРОБИРОВАЛОСЬ» В ЖИЗНИ. БЕЛИНСКИЙ И ЕГО ДРУЗЬЯ НЕ ТОЛЬКО ТЕОРЕТИЗИРОВАЛИ, НО И «ПРИМЕРЯЛИ» НА СЕБЕ НОВЫЕ ПОВЕДЕНЧЕСКИЕ МОДЕЛИ. ЖОРЖСАНДОВСКИЕ ИДЕИ ВОШлИ В ЛЮБОВНЫЙ БЫТ 1840-1860-Х ГГ., В КОТОРОМ ЯВНО ВАРЬИРОВАЛИСЬ МОТИВЫ ЖОРЖСАНДОВСКИХ ПРОИЗВЕДЕНИЙ (ПРЕЖДЕ ВСЕГО ЕЕ РОМАНОВ Jacques И Horace). ИМЕННО ЧЕРЕЗ ЖОРЖ САНД КАК ЗНАКОВУЮ ФИГУРУ ДЛЯ ВСЕХ «ЗАПАДНИКОВ» РАСКРЫВАЕТСЯ ПРИРОДА ИЗВЕСТНЫХ ЛЮБОВНО-СЕМЕЙНЫХ ДРАМ. СТАНОВИТСЯ ЯСНО, ПОЧЕМУ НИКОЛАЙ ОГАРЕВ ГОТОВ БЫЛ ВЗЯТЬ НА СЕБЯ ОТЦОВСТВО БУДУЩЕГО РЕБЕНКА СВОЕЙ ЖЕНЫ И СКУЛЬПТОРА СОКРАТА ВОРОБЬЕВА: ОН НЕ СЧИТАЛ МАРЬЮ ЛЬВОВНУ РАСПУТНИЦЕЙ, ВИНОВНОЙ В ПРЕЛЮБОДЕЯНИИ. И ПОСКОЛЬКУ ОБЩЕСТВО ОТКАЗЫВАЛО ЕЙ В УВАЖЕНИИ, ОН БРАЛ НА СЕБЯ ОТВЕТСТВЕННОСТЬ ЗА ЕЕ СПОКОЙСТВИЕ. В ЭТОМ СЛУЧАЕ ДЕЙСТВОВАЛА МОДЕЛЬ САМОУСТРАНЕНИЯ МУЖА (Jacques, 1834) И ВЕРА В НРАВСТВЕННОСТЬ ГРАЖДАНСКОГО СОЮЗА, ОСНОВАННОГО НА ВЗАИМНОЙ ЛЮБВИ (ОБРАЗЕЦ КОТОРОГО ЖОРЖ САНД ДАЛА В Нorace (1841), НА ПРИМЕРЕ ВЗАИМООТНОШЕНИЙ ЭЖЕНИ И ТЕОФИЛЯ). И В РОМАНАХ САНД, И В РУССКОМ ЛИТЕРАТУРНОМ БЫТУ ПОДОБНЫЕ СПОСОБЫ РАЗРЕШЕНИЯ ЛЮБОВНЫХ КОНФЛИКТОВ БЫЛИ СЛЕДСТВИЕМ ЧРЕЗВЫЧАЙНОЙ 
ТРУДНОСТИ, ПОЧТИ НЕВОЗМОЖНОСТИ ОФОРМЛЕНИЯ РАЗВОДА (ТЕМ БОЛЕЕ С БЛАГОПРИЯТНЫМИ ПОСЛЕДСТВИЯМИ ДЛЯ ЖЕНЩИНЫ). Т.Е. В КОНЕЧНОМ ИТОГЕ ЭТО БЫЛА ФОРМА ПРОТЕСТА ПРОТИВ ГОСУДАРСТВЕННОГО ИНСТИТУТА БРАКА.

ПОД НЕПОСРЕДСТВЕННЫМ ВОЗДЕЙСТВИЕМ РОМАНОВ ЖОРЖ САНД ЧАСТЬ РОССИЙСКОЙ ИНТЕЛЛИГЕНЦИИ К СЕРЕДИНЕ 1840-Х ГГ. ОСОЗНАЛА НЕСПРАВЕДЛИВОСТЬ ОБЩЕСТВА И НОРМАТИВНОЙ МОРАЛИ ПО ОТНОШЕНИЮ К ЖЕНЩИНЕ. ЖОРЖ САНД ПРИ ЭТОМ «СДЕЛАЛАСЬ СИМВОЛОМ ОСВОБОЖДЕНИЯ ЖЕНЩИНЫ, ЛОЗУНГОМ БОРЬБЫ ПРОТИВ ВЕКОВОГО ГНЕТА, ПАРОЛЕМ, КОТОРЫЙ БЫЛ У ВСЕХ НА УСТАХ, ВСЕМИ ПОВТОРЯЛСЯ, ВСЕМИ КОММЕНТИРОВАЛСЯ» (БРАЗОЛЕНКО, 1908: 32). С ЭНТУЗИАЗМОМ БЫЛА ВОСПРИНЯТА МОЛОДЕЖЬЮ 1840-Х ГГ. ЖОРЖСАНДОВСКАЯ КОНЦЕПЦИЯ ЛЮБВИ. «В ТО ВРЕМЯ, КАК ОТЦЫ И МАТЕРИ НЕГОДОВАЛИ НА ЖОРЖ ЗАНД, СЫНОВЬЯ И ДОЧЕРИ С ЖАДНОСТЬЮ ЧИТАЛИ ЕЕ, УВЛЕКАЯСЬ ЕЕ ХУДОЖЕСТВЕННЫМИ ОБРАЗЦАМИ, ЕЕ ТЕПЛЫМ ЧУВСТВОМ И СВЕТЛЫМИ ИДЕЯМИ.» (ШАШКОВ, 1879: 267)

\section{Авдотья Панаева в ситуации любовного треугольника}

11 ЖОРЖ САНД ДЕЙСТВИТЕЛЬНО БЫЛА, ЕСЛИ НЕ КУМИРОМ, ТО, БЕССПОРНО, ЗНАЧИТЕЛЬНЫМ ЛИТЕРАТУРНЫМ АВТОРИТЕТОМ ДЛЯ А.ПАНАЕВОЙ. ЭТО ВПОЛНЕ ЗАКОНОМЕРНО: ВЕДЬ ИМЕННО ЕЕ МУЖ, СВОИМИ ПЕРЕВОДАМИ ИЗ РОМАНА Spiridion И ДРУГИХ САНДОВСКИХ ПРОИЗВЕДЕНИЙ СУМЕЛ КАРДИНАЛЬНО ИЗМЕНИТЬ МНЕНИЕ БЕЛИНСКОГО О ФРАНЦУЗСКОЙ ПИСАТЕЛЬНИЦЕ В НАЧАЛЕ 1840-Х ГГ. ДО СВОЕГО ОСНОВАТЕЛЬНОГО ЗНАКОМСТВА С ЕЕ СОЧИНЕНИЯМИ В ОРИГИНАЛЕ, КРИТИК ПРЕНЕБРЕГАЛ ИМИ. ОН, ПО ВОСПОМИНАНИЯМ САМОЙ ПАНАЕВОЙ, «ТВЕРДИЛ ЕЙ, ПРИХОДЯ В ГОСТИ: “ГОРАЗДО БЫЛО БЫ ЛУЧШЕ ИГРАТЬ С НАМИ В ПРЕФЕРАНС, ЧЕМ ВСЕ ЧИТАТЬ ВАШУ ЖОРЖ ЗАНД”» (ПАНАЕВА, 1986: 92).

ЭТО ЗАМЕЧАНИЕ БЕЛИНСКОГО ДАЕТ ПОЧУВСТВОВАТЬ, ЧТО ПОДОБНОЕ ЧТЕНИЕ БЫЛО ДЛЯ МОЛОДОЙ ЖЕНЩИНЫ ПРИВЫЧНЫМ И ЛЮБИМЫМ ЗАНЯТИЕМ. СВОБОДОЛЮБИВАЯ НАТУРА, ОНА МОГЛА ЧЕРПАТЬ В ПРОИЗВЕДЕНИЯХ ЖОРЖ САНД ПОДДЕРЖКУ ПРОТИВ ВСЯЧЕСКОГО РОДА НАСИЛИЯ НАД ЛИЧНОСТЬЮ.

ЖОРЖСАНДОВСКИЕ ОСНОВЫ МИРОВОСПРИЯТИЯ, ПРЕДСТАВЛЕНИЯ ОБ ИНТИМНОЙ СФЕРЕ ЧЕЛОВЕЧЕСКОГО БЫТИЯ БЫЛИ БЛИЗКИ КАРТИНЕ МИРА А. ПАНАЕВОЙ. НЕСОМНЕННО, И ЕЕ ГРАЖДАНСКИЙ СОЮЗ С Н. НЕКРАСОВЫМ ВОЗНИК ПОД ВЛИЯНИЕМ ЭТИКИ ЛЮБВИ И БРАКА, СФОРМУЛИРОВАННОЙ ФРАНЦУЗСКОЙ РОМАНИСТКОЙ. ИССЛЕДОВАТЕЛИ, СПЕЦИАЛЬНО РАЗБИРАВШИЕСЯ В ЭТОЙ ЛЮБОВНО-СЕМЕЙНОЙ КОЛЛИЗИИ, ОПРОВЕРГАЛИ ОБЫВАТЕЛЬСКОЕ МНЕНИЕ О ЛЕГКОМЫСЛИИ И ДОСТУПНОСТИ ГЛАВНОЙ ЕЕ «ВИНОВНИЦЫ». К. И. ЧУКОВСКИЙ, ИЗВЕСТНЫЙ ПИСАТЕЛЬ И КРИТИК, ИЗУЧАВШИЙ БИОГРАФИЮ ПАНАЕВОЙ И СКРУПУЛЕЗНО СОБРАВШИЙ РАЗНООБРАЗНЫЕ СВИДЕТЕЛЬСТВА СОВРЕМЕННИКОВ, СОЗДАЛ НА СТРАНИЦАХ СВОЕЙ КНИГИ «ЖЕНА ПОЭТА» (1922) ОБАЯТЕЛЬНЫЙ ОБРАЗ КРАСИВОЙ, УМНОЙ, ДОБРОЙ, ПРАВДИВОЙ И АРТИСТИЧНОЙ ЖЕНЩИНЫ. НИ О КАКОМ «БЕЗДУМНОМ АДЮЛЬТЕРЕ» МЕЖДУ ПАНАЕВОЙ И НЕКРАСОВЫМ НЕ МОЖЕТ БЫТЬ И РЕЧИ, - УТВЕРЖДАЕТ Н. Н. СКАТОВ (1994:125). НАОБОРОТ, ВЛЮБИВШИЙСЯ В НЕЕ ДВАДЦАТИШЕСТИЛЕТНИЙ НЕКРАСОВ ЧУТЬ НЕ ПОКОНЧИЛ С СОБОЙ, КОГДА ОНА ЕГО ОТВЕРГЛА. И ЕМУ ПОТРЕБОВАЛОСЬ НЕСКОЛЬКО ЛЕТ, ЧТОБЫ ДОБИТЬСЯ ВЗАИМНОСТИ, ПРИЧЕМ «ПОБЕДЕ» НЕКРАСОВА ПОМОГЛО И ПРЕНЕБРЕЖИТЕЛЬНОЕ ОТНОШЕНИЕ ИВАНА 
ПАНАЕВА К МОЛОДОЙ ЖЕНЕ, КОТОРОЕ МНОГИЕ, НАБЛЮДАВШИЕ ИХ СЕМЕЙНУЮ ЖИЗНЬ, ОТМЕЧАЛИ С САМЫХ ПЕРВЫХ ЕЕ МЕСЯЦЕВ. К. И. ЧУКОВСКИЙ РАЗМЫШЛЯЛ:

<... НУЖНО НЕ ТОМУ УДИВЛЯТЬСЯ, ЧТО ОНА В КОНЦЕ КОНЦОВ СОШЛАСЬ С НЕКРАСОВЫМ, А ТОМУ, ЧТО ОНА ТАК ДОЛГО С НИМ НЕ СХОДИЛАСЬ. ОНИ ПОЗНАКОМИЛИСЬ В 1843 Г. <...>. НО ОНА НЕ СРАЗУ УСТУПИЛА ЕГО ДОМОГАНИЯМ, А ДО СТРАННОСТИ ДОЛГО УПОРСТВОВАЛА. <...> ЭТОТ ЛЮБОВНЫЙ ПОЕДИНОК ПРОДОЛЖАЛСЯ с 1843 ГОДА ПО 1848-ОЙ. (1922: 17) ${ }^{1}$

«КЛАССИЧЕСКИЙ ТРЕУГОЛЬНИК (МУЖ, ЖЕНА, “ДРУГ СЕМЕЙСТВА”) ПРЕДСТАЛ В КОМБИНАЦИЯХ, СОВСЕМ НЕ КЛАССИЧЕСКИХ», - ЗАМЕЧАЛ ИССЛЕДОВАТЕЛЬ (СКАТОВ, 1994: 124).

ПОСЛЕ ТОГО, КАК АВДОТЬЯ ПАНАЕВА СТАЛА ГРАЖДАНСКОЙ ЖЕНОЙ НИКОЛАЯ НЕКРАСОВА, ЕЕ «ЮРИДИЧЕСКИЙ МУЖ» ИВАН ПАНАЕВ НЕ ТОЛЬКО ОСТАЛСЯ «ФАКТИЧЕСКИМ ДРУГОМ ОБОИХ», НО ПРОЖИВАЛ С НИМИ В ОДНОМ ДОМЕ, НА ОДНОМ ЭТАЖЕ, А ТАКЖЕ ДО КОНЦА ЖИЗНИ (1860) ОСТАВАЛСЯ СОРЕДАКТОРОМ И ДЕЯТЕЛЬНЫМ УЧАСТНИКОМ «СОВРЕМЕННИКА». ТАКОЕ «МИРНОЕ» РАЗРЕШЕНИЕ ЛЮБОВНОЙ КОЛЛИЗИИ ОБЪЯСНЯЛОСЬ, КОНЕЧНО, НЕ ТОЛЬКО «БОЖЕСТВЕННЫМ ЛЕГКОМЫСЛИЕМ» ПАНАЕВА, НО И ОРГАНИЧНЫМ УСВОЕНИЕМ ПРИНЦИПОВ НОВОЙ НРАВСТВЕННОСТИ ВСЕМИ ТРЕМЯ ЕЕ УЧАСТНИКАМИ.

ИЗ-ЗА ОТСУТСТВИЯ ДОСТОВЕРНЫХ МАТЕРИАЛОВ ИСПОВЕДАЛЬНОГО ХАРАКТЕРА - ПИСЕМ, ДНЕВНИКОВ, ОСВЕЩАЮЩИХ ПСИХОЛОГИЮ КАЖДОГО ИЗ ПЕРСОНАЖЕЙ», МОЖНО ЛИШЬ С ИЗВЕСТНОЙ ДОЛЕЙ ДОМЫСЛА ПОПЫТАТЬСЯ ЕЕ РЕКОНСТРУИРОВАТЬ. НО ОДНО НЕСОМНЕННО: ПОЧИТАЯ «КУЛЬТ» ЖОРЖ САНД (ПАНАЕВ И ЕГО МОЛОДАЯ ЖЕНА ЕЩЕ РАНЬШЕ БЕЛИНСКОГО УВЛЕКЛИСЬ ИДЕЯМИ ФРАНЦУЗСКОЙ РОМАНИСТКИ), МНОГОЕ ДЕЛАЯ ДЛЯ ЕГО УТВЕРЖДЕНИЯ В ЖУРНАЛЕ «ОТЕЧЕСТВЕННЫЕ ЗАПИСКИ», А ПОТОМ И В «СОВРЕМЕННИКЕ», ВСЕ ТРОЕ ДОЛЖНЫ БЫЛИ ВПОЛНЕ РАЗДЕЛЯТЬ МЫСЛЬ О «СВЯТОСТИ» ЛЮБВИ И ГНУСНОСТИ БРАКА, НЕ ПРОНИКНУТОГО ЭТИМ ЧУВСТВОМ. ПРИ ТАКИХ НРАВСТВЕННЫХ УБЕЖДЕНИЯХ ВЛЮБЛЕННЫЙ НЕКРАСОВ НЕ МОГ ЧУВСТВОВАТЬ СЕБЯ БЕСЧЕСТНЫМ, ДОБИВАЯСЬ ЖЕНЫ СВОЕГО ПРИЯТЕЛЯ (ИЛИ ДАЖЕ ДРУГА?), ТЕМ БОЛЕЕ, ЧТО ОН НАБЛЮДАЛ СТРАННОСТИ СУПРУЖЕСКИХ ОТНОШЕНИЙ ПАНАЕВЫХ ВБЛИЗИ. А ПАНАЕВ ДЛЯ ТОГО И ОСТАЛСЯ ЖИТЬ ЧУТЬ ЛИ НЕ В ОДНОЙ КВАРТИРЕ С НЕКРАСОВЫМ И СВОЕЙ БЫВШЕЙ ЖЕНОЙ, ЧТОБЫ СВОИМ ПРИСУТСТВИЕМ ЗАЩИЩАТЬ ЕЕ ОТ ОБЩЕСТВЕННОГО ОСУЖДЕНИЯ (ТАК ЖЕ ТОЧНО ПОСТУПАЛ И ОГАРЕВ В ОТНОШЕНИИ К РАЗЛЮБИВШЕЙ ЕГО МАРЬЕ ЛЬВОВНЕ).

СКОРЕЕ ВСЕГО, НАСТОЙЧИВАЯ И УПОРНАЯ СТРАСТЬ МОЛОДОГО НИКОЛАЯ НЕКРАСОВА ПРОБУДИЛА ОТВЕТНОЕ ЧУВСТВО В АВДОТЬЕ ЯКОВЛЕВНЕ. С ТОЧКИ ЗРЕНИЯ ОКРУЖАЮЩИХ, СОЕДИНЯЯСЬ С НЕКРАСОВЫМ, ОНА СОВЕРШАЛА ЧУТЬ ЛИ НЕ АКТ САМОПОЖЕРТВОВАНИЯ «ВЕДЬ ПАНАЕВ БЫЛ ДЕНДИ, А НЕКРАСОВ ТЕМНЫЙ ПРОХОДИМЕЦ!» - ТАКОВО БЫЛО МНЕНИЕ СВЕТА (ЧУКОВСКИЙ, 1922: 18-19). ОДИН ИЗ СОВРЕМЕННИКОВ СВИДЕТЕЛЬСТВОВАЛ: «ПАНАЕВ БЫЛ ЧЕЛОВЕК ИСТИННО ПОРЯДОЧНЫЙ, ПОРЯДОЧНОЙ ФАМИЛИИ И ПОРЯДОЧНЫХ СВЯЗЕЙ; НАРУЖНОСТЬ ЕГО БЫЛА ВЕСЬМА КРАСИВАЯ И СИМПАТИЧНАЯ, ТОГДА КАК НЕКРАСОВ ИМЕЛ ВИД ИСТИННОГО БРОДЯГИ И ПО НАРУЖНОСТИ, И ПО ОБЩЕСТВЕННОМУ ПОЛОЖЕНИЮ ${ }^{2}$ „» ИСТОРИК Т. Н. ГРАНОВСКИЙ, ЦЕНИВШИЙ УМ И ОБАЯНИЕ АВДОТЬИ ПАНАЕВОЙ, СЧИТАЛ ЕЕ СВЯЗЬ С НЕКРАСОВЫМ НЕСЧАСТЬЕМ ДЛЯ НЕЕ. «ЖАЛЬ ЭТОЙ БЕДНОЙ ЖЕНЩИНЫ, - ПИСАЛ ОН ЖЕНЕ 11 ЯНВАРЯ 1851 Г. - В НЕЙ ДО СИХ ПОР МНОГО УМА И ДОБРОТЫ ИСТИННОЙ, НО ЧТО ЗА ПОНЯТИЯ! ВИДНО, ЧТО НАД НЕЮ ТЯГОТЕЕТ ГРУБОЕ ВЛИЯНИЕ НЕОБРАЗОВАННОГО, ПОШЛОГО СЕРДЦЕМ ЧЕЛОВЕКА ${ }^{3}$ „» ЭТОТ МОТИВ ЖАЛОСТИ К ПРЕКРАСНОЙ ЖЕНЩИНЕ, ПОГУБЛЕННОЙ 
НЕДОСТОЙНЫМ МУЖЧИНОЙ, ПРОХОДИТ ЧЕРЕЗ ВСЕ ПИСЬМА ГРАНОВСКОГО, НАПИСАННЫЕ ПОД ВПЕЧАТЛЕНИЕМ ВСТРЕЧ С НЕЙ. «ЖАЛЬ БЕДНОЙ ЖЕНЩИНЫ! - СООБЩАЛ ОН ЖЕНЕ В 1854 Г. - СКОЛЬКО В НЕЙ ХОРОШЕГО. А МИР, ЕЕ ОКРУЖАЮЩИЙ, В СОСТОЯНИИ ЗАДАВИТЬ КОГО ХОЧЕШЬ ${ }^{4}$.» «А КАК ЖАЛЬ ЕЕ. ОНА ПОХУДЕЛА, ПОДУРНЕЛА И ОЧЕНЬ ГРУСТНА», - ПОВТОРЯЛ ОН 30 АПРЕЛЯ 1855 Г. ${ }^{5}$.

17 МОЖНО ПРЕДПОЛОЖИТЬ, ЧТО ПОНАЧАЛУ САМА ПАНАЕВА ВИДЕЛА В СВОЕМ ГРАЖДАНСКОМ БРАКЕ С НЕКРАСОВЫМ АНАЛОГИЮ С ИДЕАЛЬНЫМИ СОЮЗАМИ, ИЗОБРАЖЕННЫМИ ЖОРЖ САНД В РОМАНЕ Horace (1841). ТЕОФИЛЯ И ЭЖЕНИ, МАРТУ И АРСЕНА СВЯЗЫВАЛА НЕ ТОЛЬКО И НЕ СТОЛЬКО СТРАСТЬ, СКОЛЬКО ВЗАИМНОЕ УВАЖЕНИЕ, ОБЩНОСТЬ ДУХОВНЫХ ИНТЕРЕСОВ, МИРОВОСПРИЯТИЯ. ПЕРВОЕ ВРЕМЯ НЕКРАСОВА И ПАНАЕВУ ДЕЙСТВИТЕЛЬНО СОЕДИНЯЛИ ВЗАИМНАЯ ЛЮБОВЬ, ОБЩИЕ ВЗГЛЯДЫ НА ЛИТЕРАТУРУ, ЗАДАЧИ ЖУРНАЛЬНОЙ РАБОТЫ, ТВОРЧЕСТВО. В СУДЬБЕ АВДОТЬИ ПАНАЕВОЙ ЛИЧНОЕ И ПУБЛИЧНОЕ ТЕСНО ПЕРЕПЛЕЛИСЬ. КОГДА ЦЕНЗУРА В 1848 Г. ЗАПРЕТИЛА ВСЕ ПЕРЕВОДНЫЕ РОМАНЫ (В ТОМ ЧИСЛЕ Piccinino И Leone Leoni ЖОРЖ САНД), ПРЕДНАЗНАЧЕННЫЕ НЕКРАСОВЫМ ДЛЯ «СОВРЕМЕННИКА», ОН, ЧТОБЫ ПОДДЕРЖАТЬ ИЗДАНИЕ, ПРИВЛЕК СВОЮ ПОДРУГУ К НАПИСАНИЮ СОВМЕСТНОГО БОЛЬШОГО РОМАНА «С ПРОДОЛЖЕНИЕМ». ТАК ПОЯВИЛИСЬ «ТРИ СТРАНЫ СВЕТА» (1848-1849), ЗАНИМАТЕЛЬНОЕ ЖУРНАЛЬНОЕ ЧТЕНИЕ, РАССЧИТАННОЕ «НЕ НА ТОНКИХ ЗНАТОКОВ, А НА МАССУ, <...> В СВОИХ ВКУСАХ НЕ ВЕСЬМА ТРЕБОВАТЕЛЬНУЮ» (ПЫПИН, 1905: 154). ПРИ ЭТОМ ПАНАЕВА «ОТВЕЧАЛА» ЗА ЛЮБОВНЫЙ СЮЖЕТ КАК В ЭТОМ, ТАК И В СЛЕДУЮЩЕМ, НАПИСАННОМ ТОЖЕ В СОАВТОРСТВЕ С НЕКРАСОВЫМ РОМАНЕ - «МЕРТВОЕ ОЗЕРО» (1851). НО БОЛЕЕ ИНТЕРЕСНЫМИ И ЗНАЧИТЕЛЬНЫМИ БЫЛИ САМОСТОЯТЕЛЬНО СОЗДАННЫЕ ЕЮ ПОВЕСТИ И РАССКАЗЫ, ПУБЛИКОВАВШИЕСЯ В «СОВРЕМЕННИКЕ» С КОНЦА 1840-Х ГГ. ТАКИМ ОБРАЗОМ, «МЕДОВЫЕ МЕСЯЦЫ» ПАНАЕВОЙ И НЕКРАСОВА «ПРОТЕКАЛИ В ХЛОПОТЛИВОЙ РАБОТЕ» (ЧУКОВСКИЙ, 1922: 18). ПЕРВЫЙ ИЗ РОМАНОВ ИМЕЛ БОЛЬШОЙ ЧИТАТЕЛЬСКИЙ УСПЕХ И ЗНАЧИТЕЛЬНО УВЕЛИЧИЛ ЧИСЛО ПОДПИСЧИКОВ «СОВРЕМЕННИКА», ДЛЯ КОТОРОГО ОН И БЫЛ НАПИСАН. ДЕЛА ЖУРНАЛА ПАНАЕВА ПРИНИМАЛА БЛИЗКО К СЕРДЦУ. В ХОЗЯЙСТВЕННОМ И ДЕЛОВОМ ОТНОШЕНИИ ОНА ОКАЗАЛАСЬ «КЛАДОМ» ДЛЯ НЕКРАСОВА. «ОНА ЧИТАЛА РУКОПИСИ, ДЕРЖАЛА КОРРЕКТУРЫ, ПРИКАРМЛИВАЛА НУЖНЫХ СОТРУДНИКОВ.» (ТАМ ЖЕ: 20) ОНА ДО ТОНКОСТИ ПОСТИГЛА СТИЛЬ РАЗНЫХ ОБЕДОВ, ДАВАЕМЫХ НЕКРАСОВЫМ В РЕДАКЦИИ «СОВРЕМЕННИКА». ВЫЙДЯ ИЗ АКТЕРСКОЙ СЕМЬИ, ОНА АРТИСТИЧНО ИГРАЛА ВСЕ РОЛИ: С СЕМИНАРИСТАМИ БЫЛА «ДЕМОКРАТИЧЕСКИ ПРОСТА», С ГЕНЕРАЛАМИ — «ВЕЛИКОСВЕТСКАЯ БАРЫНЯ» (ТАМ ЖЕ).

МОЖНО УТВЕРЖДАТЬ, ЧТО ЧЕРЕЗ СВОИХ ЗНАМЕНИТЫХ МУЖЕЙ - ПАНАЕВА И НЕКРАСОВА - АВДОТЬЯ ПАНАЕВА ВОШЛА В КРУГ САМЫХ СВЕТЛЫХ УМОВ И ЗАМЕЧАТЕЛЬНЫХ РОССИЙСКИХ ТАЛАНТОВ, БЫЛА НЕ ТОЛЬКО ХОРОШЕЙ ХОЗЯЙКОЙ И УСТРОИТЕЛЬНИЦЕЙ ОБЕДОВ В РЕДАКЦИИ «СОВРЕМЕННИКА», НО И ДЕЯТЕЛЬНЫМ СОТРУДНИКОМ ЭТОГО ЖУРНАЛА.

И, ТЕМ НЕ МЕНЕЕ, ВСКОРЕ СОВМЕСТНАЯ ЖИЗНЬ ПАНАЕВОЙ И НЕКРАСОВА ПРЕВРАТИЛАСЬ В АД. ГАРМОНИЧНОГО СОЮЗА НЕ ПОЛУЧИЛОСЬ, И, СУДЯ ПО ВСЕМУ, НЕ ПО ВИНЕ ЖЕНЩИНЫ. НЕКРАСОВ ЯВНО «НЕ ДОТЯГИВАЛ» ДО УРОВНЯ ИДЕАЛЬНОГО ГЕРОЯ, СПОСОБНОГО ДАТЬ СЧАСТЬЕ И СПОКОЙСТВИЕ СВОЕЙ ПОДРУГЕ В СЛОЖНОЙ «НЕЗАКОННОЙ» СИТУАЦИИ. «ОН ЛЮБИЛ ЕЕ УГРЮМОЙ, РЕВНИВОЙ, ИЗНУРИТЕЛЬНО ТРУДНОЙ ЛЮБОВЬЮ», - ЗАМЕЧАЛ К. И. ЧУКОВСКИЙ (ТАМ ЖЕ: 11). ОЧЕВИДЦЫ ПРИВОДЯТ УБЕДИТЕЛЬНЫЕ ФАКТЫ НЕДОСТОЙНОГО 
ПОВЕДЕНИЯ НЕКРАСОВА. «САМОИСТЯЗАТЕЛЬ, КАЖДОЕ СВОЕ ЧУВСТВО ПРЕВРАЩАЮЩИЙ В КАЗНЬ, ОН И ЛЮБИТЬ УМЕЛ ТОЛЬКО МУЧЕНИЧЕСКИ, ТОЛЬКО МУЧИТЕЛЬСКИ», - ЗАКЛЮЧАЛ БИОГРАФ ПАНАЕВОЙ (ТАМ ЖЕ: 13).

СОВРЕМЕННЫЙ ЛИТЕРАТУРОВЕД Н.Н. СКАТОВ ОБЪЯСНИЛ МРАЧНЫЙ, ТРАГИЧЕСКИЙ ХАРАКТЕР ЛЮБВИ НЕКРАСОВА БОЛЕЗНЕННЫМ ЧУВСТВОМ «НЕСОСТОЯВШЕГОСЯ ОТЦОВСТВА» (1994: 130-133). ВОЗМОЖНО, ВСЯ ЛЮБОВНО-СЕМЕЙНАЯ ДРАМА ПАНАЕВА, НЕКРАСОВА И ПАНАЕВОЙ РАЗВИВАЛАСЬ БЫ ИНАЧЕ, ЕСЛИ БЫ ЖИВЫМИ БЫЛИ РОЖДЕННЫЕ ОТ ПЕРВОГО И ВТОРОГО СОЮЗОВ ДЕТИ. ИЗВЕСТНО, ЧТО ИВАН ПАНАЕВ, НЕЗАДОЛГО ДО СВОЕЙ СМЕРТИ, УГОВАРИВАЛ СВОЮ БЫВШУЮ ЖЕНУ ПОКИНУТЬ ПЕТЕРБУРГ И ПОСЕЛИТЬСЯ ВМЕСТЕ С НИМ В ДЕРЕВНЕ И ОЧЕНЬ ОБРАДОВАЛСЯ ЕЕ СОГЛАСИЮ. ОН УМЕР НА ЕЕ РУКАХ, ПРОСЯ О ПРОЩЕНИИ (ПАНАЕВА, 1986: 314-316). А ЭПИЛОГОМ ЭТОЙ ПОЧТИ РОМАНИЧЕСКОЙ ИСТОРИИ СТАЛ НЕОЖИДАННЫЙ ДЛЯ НЕКРАСОВА «ЗАКОННЫЙ» БРАК ПАНАЕВОЙ С ЕГО СЕКРЕТАРЕМ А. Ф. ГОЛОВАЧЕВЫМ, ПРИНЕСШИЙ ЕЙ ДОЛГОЖДАННОЕ МАТЕРИНСТВО.

\section{Принципы сюжетостроения и мотивной организации прозы А. Я. Панаевой}

УЖЕ В ДЕБЮТНОМ СОЧИНЕНИИ А. ПАНАЕВОЙ, ПОВЕСТИ «СЕМЕЙСТВО ТАЛЬНИКОВЫХ» (1847), ОТРАЗИЛАСЬ ХАРАКТЕРНАЯ ДЛЯ ПОЭТИКИ ЖЕНСКОЙ ЛИТЕРАТУРЫ ТЕНДЕНЦИЯ - ОБРАЩЕНИЕ К ПРОБЛЕМАМ ВНУТРИСЕМЕЙНЫХ СВЯЗЕЙ, ОТНОШЕНИЙ МЕЖДУ ДЕТЬМИ И РОДИТЕЛЯМИ. В ЖЕНСКОЙ ПРОЗЕ, СОСРЕДОТОЧЕННОЙ НА «МАЛОМ», ИНТИМНОМ МИРЕ, СЕМЬЯ РАССМАТРИВАЕТСЯ КАК МИКРОКОСМ, ГДЕ ФОРМИРУЮТСЯ МОДЕЛИ МЕЖЛИЧНОСТНЫХ ОТНОШЕНИЙ, КОТОРЫЕ ПОЛУЧАЮТ РЕАЛИЗАЦИЮ В МАКРОКОСМЕ (СОЦИУМЕ).

НАСТРАДАВШИСЬ В ДЕТСТВЕ И ОТРОЧЕСТВЕ ОТ ПРИТЕСНЕНИЙ МАТЕРИ, ОНА КОЛОРИТНО ОПИСАЛА СЕМЕЙНЫЙ ГНЕТ И ДЕСПОТИЗМ. НАПИСАННОЕ ОТ ЛИЦА ДЕВУШКИ НАТАШИ И ПРЕДСТАВЛЕННОЕ В ВИДЕ «ЗАПИСОК, НАЙДЕННЫХ В БУМАГАХ ПОКОЙНИЦЫ», ПРОИЗВЕДЕНИЕ ВОССОЗДАВАЛО ПРОЦЕСС ФОРМИРОВАНИЯ ЖЕНСКОЙ ЛИЧНОСТИ В УСЛОВИЯХ ПРЕНЕБРЕЖЕНИЯ К ЕЕ ЭЛЕМЕНТАРНЫМ ДУХОВНЫМ ЗАПРОСАМ. ПО СЛОВАМ КОРНЕЯ ЧУКОВСКОГО, «ВСЯ СИСТЕМА ТОГДАШНЕГО ВОСПИТАНИЯ, ТЕСНО СВЯЗАННАЯ С КРЕПОСТНИЧЕСКИМ <...> СТРОЕМ <...>, ЗДЕСЬ БЫЛА ОБЛИЧЕНА И ОПОЗОРЕНА» (1928: 97). ВПОЛНЕ ПОНЯТНО, ЧТО СЕКРЕТНЫЙ ЦЕНЗУРНЫЙ КОМИТЕТ, УСМОТРЕВ В СОЧИНЕНИИ «РЕВОЛЮЦИОННОЕ ПОТРЯСЕНИЕ СЕМЕЙНЫХ ОСНОВ», ЗАПРЕТИЛ ЕГО ПУБЛИКАЦИЮ. АПРЕДСЕДАТЕЛЬ КОМИТЕТА ГРАФ БУТУРЛИН СОБСТВЕННОРУЧНО НАПИСАЛ В ЗАКЛЮЧЕНИЕ: «НЕ ПОЗВОЛЯЮ ЗА БЕЗНРАВСТВЕННОСТЬ И ПОДРЫВ РОДИТЕЛЬСКОЙ ВЛАСТИ.» (ТАМ ЖЕ)

И ХОТЯ В СТРОГОМ И РЕЗКОМ ИЗОБРАЖЕНИИ ВСЕГО ГРУБОГО И ЖЕСТОКОГО В ДОМАШНЕМ ВОСПИТАНИИ ГЕРОИНИ, НЕСОМНЕННО, ЧУВСТВОВАЛАСЬ «РУКА» НЕКРАСОВА, ОБЛИЧИТЕЛЬНЫЙ ПАФОС БЫЛ ПРИСУЩ И ВСЕМ ПОСЛЕДУЮЩИМ ПРОИЗВЕДЕНИЯМ ПАНАЕВОЙ. В ОТЛИЧИЕ ОТ ДРУГИХ ПИСАТЕЛЬНИЦ-СОВРЕМЕННИЦ, ОНА ВЗЯЛА СЕБЕ, ПОДОБНО ЖОРЖ САНД, МУЖСКОЙ ПСЕВДОНИМ - СТАНИЦКИЙ. ВОЗМОЖНО, В ТАКОМ ВЫБОРЕ СКАЗАЛОСЬ СТРЕМЛЕНИЕ ПРЕОДОЛЕТЬ ОДНОСТОРОННОСТЬ «ЖЕНСКОГО» ВИДЕНИЯ, А, МОЖЕТ БЫТЬ, ЕЙ ХОТЕЛОСЬ ОРГАНИЧНО ВЛИТЬСЯ В РЯДЫ ЛИТЕРАТОРОВМУЖЧИН, СТОЯВШИХ У ИСТОКОВ НОВОГО НАПРАВЛЕНИЯ «СОВРЕМЕННИКА». ПО- 
ВИДИМОМУ, ИНИЦИАТИВА ПИСАТЬ ПОД ЧУЖИМ ИМЕНЕМ ИСХОДИЛА ОТ САМОЙ ПАНАЕВОЙ. ОНА ОТКАЗАЛАСЬ ПОДПИСЫВАТЬ СВОИ ПРОИЗВЕДЕНИЯ ФАМИЛИЕЙ МУЖА, ЖЕЛАЯ ТЕМ САМЫМ ОТСТРАНИТЬСЯ ОТ ЕГО ЛИТЕРАТУРНОЙ СЛАВЫ И ОТ ДОПОЛНИТЕЛЬНОГО МУЖСКОГО ВЛИЯНИЯ, А ТАКЖЕ УТВЕРДИТЬ ЗНАЧИМОСТЬ СОБСТВЕННЫХ НАБЛЮДЕНИЙ И ОПРОВЕРГНУТЬ УКОРЕНИВШИЕСЯ ВЗГЛЯДЫ О ТВОРЧЕСКОЙ И ИНТЕЛЛЕКТУАЛЬНОЙ НЕПОЛНОЦЕННОСТИ ЖЕНЩИНЫ. НАКОНЕЦ, МУЖСКАЯ «МАСКА» МОГЛА БЫТЬ И ЗНАКОМ ПРЯМОГО ПОДРАЖАНИЯ ЖОРЖ САНД. ХОРОШО ЗНАЯ И РАЗДЕЛЯЯ ИНТЕРЕСЫ КРУЖКА БЕЛИНСКОГО, ОНА ПОНИМАЛА, ПОЧЕМУ В НЕМ ВОЗНИК «КУЛЬТ» ФРАНЦУЗСКОЙ РОМАНИСТКИ, ОБУСЛОВИВШИЙ ВЫБОР МНОГИХ МАТЕРИАЛОВ ВНАЧАЛЕ ДЛЯ «ОТЕЧЕСТВЕННЫХ ЗАПИСОК», А ЗАТЕМ И ДЛЯ «СОВРЕМЕННИКА».

УЖЕ ПЕРВОЕ ПРОИЗВЕДЕНИЕ А. ПАНАЕВОЙ ПОБУДИЛО САТИРИКА Н. Ф. ЩЕРБИНУ НАПИСАТЬ НА НЕЕ ЗЛУЮ ЭПИГРАММУ, В КОТОРОЙ ВЫСМЕИВАЛИСЬ ЕЕ ПРЕТЕНЗИИ УПОДОБИТЬСЯ ПРОСЛАВЛЕННОЙ ЗНАМЕНИТОСТИ. В «СОННИКЕ СОВРЕМЕННОЙ РУССКОЙ ЛИТЕРАТУРЫ» ЩЕРБИНА УТВЕРЖДАЛ : «СТАНИЦКОГО (АВДОТЬЮ) ВО СНЕ ВИДЕТЬ ПРЕДВЕЩАЕТ ОТЦА И МАТЬ В ГРЯЗЬ ВТОПТАТЬ - ЛИШЬ БЫ ТОЛЬКО ПЛОХУЮ ПОВЕСТУШКУ НАПИСАТЬ ИЛИ ЖЕ УВИДЕТЬ, КАК КОМИЧЕСКИ РУССКАЯ ХОЛОПКА КОРЧИТ ИЗ СЕБЯ ЭМАНЦИПИРОВАННУЮ ЖОРЖ САНД.» (1929: 148-149)

НО, НЕСМОТРЯ НА МУЖСКОЙ ПСЕВДОНИМ, «ЖЕНСКАЯ» ПРИРОДА МИРОВОСПРИЯТИЯ ОБНАРУЖИЛАСЬ УЖЕ В САМЫХ РАННИХ СОЧИНЕНИЯХ ПАНАЕВОЙ. ПРОЗА ПАНАЕВОЙ ВЫРАЖАЕТ ОСОБЕННОСТИ ЖЕНСКОГО МИРОВИДЕНИЯ, СТРУКТУРНО ВОПЛОЩЕННОГО ПРЕЖДЕ ВСЕГО В ПОЭТИКЕ СЮЖЕТА. А. ПАНАЕВА ИСПОЛЬЗУЕТ РАСПРОСТРАНЕННЫЙ В РУССКОЙ ЛИТЕРАТУРЕ 1840-1850-Х ГГ. ПРИНЦИП ДВУХЧАСТНОГО СТРУКТУРИРОВАНИЯ СЮЖЕТА. В ОТЛИЧИЕ ОТ АВТОРОВ-МУЖЧИН (И. ПАНАЕВА, А.ДРУЖИНИНА, А. ПИСЕМСКОГО, И. ТУРГЕНЕВА), КОТОРЫЕ СВЯЗЫВАЛИ ДВУХЧАСТНУЮ СЮЖЕТНУЮ МОДЕЛЬ С МОТИВОМ ПИГМАЛИОНА И ГАЛАТЕИ (В ПЕРВОЙ ЧАСТИ ОБЫЧНО ИЗОБРАЖАЕТСЯ БЕЗРАДОСТНОЕ СУЩЕСТВОВАНИЕ ДЕВУШКИ В ЧУЖДОЙ ЕЙ ПО ДУХУ СРЕДЕ; ВО ВТОРОЙ - ПОВЕСТВУЕТСЯ О ВСТРЕЧЕ ГЕРОИНИ С «ДЕЯТЕЛЬНЫМ ЧЕЛОВЕКОМ», ПОД ВЛИЯНИЕМ КОТОРОГО ОНА, КАК СПЯЩАЯ КРАСАВИЦА, ПРОБУЖДАЕТСЯ ОТ СНА), У А. ПАНАЕВОЙ ОНА ОБУСЛОВЛЕНА МОТИВОМ СВОБОДЫ ВЫБОРА И ВОЗДАЯНИЯ, ЯВЛЯЮЩЕГОСЯ ДОМИНАНТНЫМ В СЮЖЕТНОЙ ОРГАНИЗАЦИИ ЕЕ ПРОЗЫ.

ОСМЫСЛЕНИЕ ЛЮБВИ И БРАКА КАК ГЛАВНЫХ СОБЫТИЙ В ЖИЗНИ ЖЕНЩИНЫ СТАЛО ТЕМОЙ ЕЕ ПОВЕСТИ «БЕЗОБРАЗНЫЙ МУЖ» (1848). В НЕЙ ОНА ИЗБРАЛА ПОПУЛЯРНУЮ ФОРМУ ЭПИСТОЛЯРНОЙ ИСПОВЕДИ ГЕРОИНИ К ПОДРУГЕ ИЗ ПЕТЕРБУРГА. НА ПРОТЯЖЕНИИ ГОДА ПЕРЕПИСКИ ПРОИЗОШЛО СТРЕМИТЕЛЬНОЕ ВЗРОСЛЕНИЕ, РАЗВИТИЕ НАИВНОЙ ДЕВУШКИ, УСТАВШЕЙ ОТ ОТЦОВСКИХ ПРИТЕСНЕНИЙ И ЖАЖДУЩЕЙ ЛАСКИ И ТЕПЛА, В УМУДРЕННУЮ ГОРЕМ ЖЕНЩИНУ, КОТОРАЯ РАСПЛАЧИВАЛАСЬ ЗА ПОПЫТКУ ОБРЕСТИ СЧАСТЬЕ В БРАКЕ ПО РАСЧЕТУ. ВСЛЕД ЗА Е. А. ГАН, М. С. ЖУКОВОЙ ${ }^{6}$ И П. Н. КУДРЯВЦЕВЫМ , ПАНАЕВА ПРИВОДИЛА СВОЮ ГЕРОИНЮ К ОТКРЫТИЮ УЖАСОВ ИНТИМНОЙ ЖИЗНИ С МУЖЧИНОЙ, КОТОРЫЙ ВНУШАЕТ ЕЙ ОТВРАЩЕНИЕ. «У НАС, НЕСЧАСТНЫХ ДЕВУШЕК, - ПРИЗНАВАЛАСЬ ОНА, - БЫВАЮТ КАКИЕ-ТО ОСОБЕННЫЕ ПОНЯТИЯ О ЛЮДЯХ, О ЖИЗНИ, О ЗАМУЖЕСТВЕ. Я, НАПРИМЕР, ДУМАЛА, ЧТО БУДУ ЛЮБИТЬ МУЖА - И ОН БУДЕТ МЕНЯ ЛАСКАТЬ, А ЕСЛИ НЕ ПОЛЮБЛЮ, ТАК ОН НЕ ПОСМЕЕТ ДОКУЧАТЬ МНЕ СВОИМИ ЛАСКАМИ И ТРЕБОВАТЬ ИХ.» (СТАНИЦКИЙ, 1848: 147) С КАЖДЫМ НОВЫМ ПОСЛАНИЕМ ЭТО ПОНАЧАЛУ ЛЕГКОМЫСЛЕННОЕ СУЩЕСТВО «УМНЕЛО», НАБИРАЛОСЬ ОПЫТА В СТРАДАНИЯХ. РАНЕЕ ТАК ЗАВИДОВАВШАЯ БОГАТОЙ ПОДРУГЕ ГЕРОИНЯ ОТКРЫЛА, ЧТО ЩОДНИ ДЕНЬГИ ТАКЖЕ 
НЕ МОГУТ ДОСТАВИТЬ СЧАСТЬЯ ЖЕНЩИНЕ». ОТ ЛАСК БЕЗОБРАЗНОГО МУЖА ОНА «СОДРОГАЕТСЯ», НО ДОЛЖНА ТЕРПЕТЬ ИХ, ПОТОМУ ЧТО ПРЕВРАТИЛАСЬ «В ЕГО ПРИНАДЛЕЖНОСТЬ, <...> ПРОДАЛА СЕБЯ ЕМУ... » И ПРИШЛО ЗАПОЗДАЛОЕ РАСКАЯНИЕ: «ЕСТЬ ВЕЩИ, КОТОРЫЕ НЕ ПОКУПАЮТСЯ И НЕ ПРОДАЮТСЯ. ЖЕНЩИНА ДОЛЖНА БЕРЕЧЬ СВОЮ СВОБОДУ, ЧТОБЫ ЛЮБИТЬ, КОГО ОНА ЗАХОЧЕТ.» (ТАМ ЖЕ: 150)

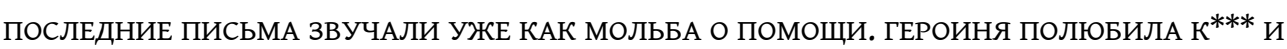
ОТКРЫЛА ЭТО, ЧИТАЯ С НИМ ВДВОЕМ «ИНДИАНУ». РОМАН ЖОРЖ САНД СТАЛ ЗНАКОМ, ПОМОГАЮЩИМ ВЛЮБЛЕННЫМ ПОНЯТЬ СЕБЯ И ДРУГ ДРУГА: «ПЕРЕСТАВ ЧИТАТЬ, Я СКАЗАЛА ЕМУ СОВЕРШЕННО БЕЗ УМЫСЛУ: “ВОТ КАК НАС ЛЮБЯТ, ВЕРЬ КОМУ-НИБУДЬ!” ОН СТРАШНО ПОБЛЕДНЕЛ И С УПРЕКОМ СКАЗАЛ: “ВЫ МОГЛИ БЫ ПОВЕРИТЬ, ВЫ САМИ СТРАДАЕТЕ.” (ТАМ ЖЕ: 156) ЗАВЕРШАЛИСЬ ЭТИ МУКИ ЖЕНЫ «БЕЗОБРАЗНОГО» МУЖА ЕЕ ТОСКЛИВЫМИ ПРЕДЧУВСТВИЯМИ ГИБЕЛИ: «МНЕ САМОЙ ПРИШЛО В ГОЛОВУ, БУДТО МОЙ МУЖ ВОЗИТ МЕНЯ МЕРТВУЮ КО ВСЕМ ЗА ПОСЛЕДНИМ ПОЦЕЛУЕМ <... ЧТО МЕНЯ ОЖИДАЕТ? ... О, ПОЩАДИТЕ, ПОЩАДИТЕ МЕНЯ!» (ТАМ ЖЕ: 158)

В ЭТОЙ НЕПРИТЯЗАТЕЛЬНОЙ ПОВЕСТИ СОДЕРЖАЛОСЬ ВЗВОЛНОВАННОЕ ПРЕДОСТЕРЕЖЕНИЕ ТЕМ ЖЕНЩИНАМ, КОТОРЫЕ МЕЧТАЛИ ДОВОЛЬСТВОМ СКРАСИТЬ БРАК БЕЗ ЛЮБВИ. СУПРУЖЕСКИЕ ОТНОШЕНИЯ, В КОТОРЫХ НЕ БЫЛО ВЗАИМНОСТИ, БЫЛИ ПРЕДСТАВЛЕНЫ КАК НЕЧТО ОТВРАТИТЕЛЬНОЕ, БЕЗНРАВСТВЕННОЕ И ПОСТЫДНОЕ.

В НЕБОЛЬШОМ РАССКАЗЕ ПАНАЕВОЙ «ЖЕНА ЧАСОВОГО МАСТЕРА» (1849) БЫЛА ПЕРЕДАНА ДРАМАТИЧЕСКАЯ ИСТОРИЯ ПРЕЛЕСТНОЙ МАТИЛЬДЫ, МУЖ КОТОРОЙ ИЗ СРЕБРОЛЮБИЯ ЗАСТАВИЛ ЕЕ СДЕЛАТЬСЯ СОДЕРЖАНКОЙ БОГАТЫХ КЛИЕНТОВ. ПОВЕСТВОВАТЕЛЬ ЯВИЛСЯ СВИДЕТЕЛЕМ СТРАДАНИЙ МОЛОДОЙ ЖЕНЩИНЫ, ЕЕ ПОСТЕПЕННОГО УГАСАНИЯ И СМЕРТИ ОТ ЧАХОТКИ. ЗАТО ЕЕ СУПРУГ, ОВДОВЕВ, ОТКРЫЛ У СЕБЯ НА РОДИНЕ В ЖЕНЕВЕ САМЫЙ РОСКОШНЫЙ МАГАЗИН ${ }^{8}$. ЭТОТ РАССКАЗ, ВЫДЕРЖАННЫЙ В ПРЕДЕЛЬНО ОБЪЕКТИВНОЙ МАНЕРЕ, НАПОМИНАЛ ФИЗИОЛОГИЧЕСКИЙ ОЧЕРК, СТОЛЬ ПОПУЛЯРНЫЙ СРЕДИ ПРЕДСТАВИТЕЛЕЙ НАТУРАЛЬНОЙ ШКОЛЫ. НО ЗА БЕССТРАСТНЫМ ПОВЕСТВОВАНИЕМ ОЩУЩАЛОСЬ ГНЕВНОЕ ВОЗМУЩЕНИЕ БЕСПРАВИЕМ ЖЕНЩИНЫ, К КОТОРОЙ МУЖ И ОБЩЕСТВО ОТНОСЯТСЯ КАК К КРАСИВОЙ ИГРУШКЕ. ПОВЕСТЬ ПАНАЕВОЙ «ПАСЕКА» (1849), НЕСОМНЕННО, БЫЛА ЗАДУМАНА С ЦЕЛЬЮ ПОРАЗМЫШЛЯТЬ О НЕПРОИЗВОЛЬНОЙ ПРИРОДЕ ЛЮБВИ, СЛОЖНОСТИ ЧЕЛОВЕЧЕСКИХ ОТНОШЕНИЙ В БРАКЕ. НЕУДАВШЕЕСЯ В ЦЕЛОМ (СЛИШКОМ МНОГО В НЕМ СЮЖЕТНЫХ ОТВЕТВЛЕНИЙ, ПЕРСОНАЖЕЙ, НЕ ПОЛУЧИВШИХ ПСИХОЛОГИЧЕСКОГО РАЗВИТИЯ) ПРОИЗВЕДЕНИЕ РАСПАДАЛОСЬ НА НЕСКОЛЬКО МАСТЕРСКИ ВЫПИСАННЫХ ЭПИЗОДОВ. К ЧИСЛУ НАИБОЛЕЕ УДАЧНЫХ ОТНОСИЛАСЬ СЦЕНА ПЕРВОГО ЗНАКОМСТВА ЧИТАТЕЛЯ С БЕЛКОЙ, ДЕВУШКОЙ-ПОДРОСТКОМ, ПРОЗВАННОЙ ТАК ЗА СВОЮ ГИБКОСТЬ, ЛОВКОСТЬ, УМЕНИЕ ЖИТЬ В ЛЕСУ КАК ДОМА. АВТОР-ЖЕНЩИНА ПРЕДЛОЖИЛА СВОЙ ВАРИАНТ «ЕСТЕСТВЕННОГО» ЧЕЛОВЕКА, ПРЕКРАСНОГО ФИЗИЧЕСКИ И НРАВСТВЕННО, СЧАСТЛИВОГО ТОЛЬКО НА ВОЛЕ. НО ОБЩЕСТВО НЕ ОСТАВИЛО В ПОКОЕ КРАСИВУЮ ДЕВУШКУ. «ПРОДАННАЯ ЕВРЕЮ» И СПАСЕННАЯ ИЗ НЕВОЛИ, ОНА ИЗ БЛАГОДАРНОСТИ ВЫШЛА ЗАМУЖ ЗА ВЕЛИКОДУШНОГО ПОРЯДОЧНОГО ЧЕЛОВЕКА, ЗЕНОВСКОГО.

32 УВАЖАЯ МУЖА И НЕ ЖЕЛАЯ ЕГО ОГОРЧАТЬ, БЕЛКА НЕОЖИДАННО ВЛЮБИЛАСЬ В ЕГО ДРУГА КАМЫШОВА, КОТОРЫЙ, В СВОЮ ОЧЕРЕДЬ, «ЗАБЫЛ О СВОЕМ НЕСЧАСТНОМ ТОВАРИЩЕ, ДАЖЕ О СЕБЕ САМОМ <...> И ТОЛЬКО ЖИЛ ЧУВСТВОМ, КОТОРОГО ОН И НЕ ПОНИМАЛ ПРЕЖДЕ ${ }^{9}$. КАЖДЫЙ ИЗ УЧАСТНИКОВ ЭТОЙ ЛЮБОВНОЙ КОЛЛИЗИИ ВЕЛ СЕБЯ БЛАГОРОДНО, А МУЖ ОТ СКРЫТЫХ СТРАДАНИЙ ДАЖЕ ТЯЖЕЛО ЗАБОЛЕЛ. ПОЧТИ ПРИ 
СМЕРТИ, ОН ДУМАЛ, ОДНАКО, ТОЛЬКО О СЧАСТЬЕ ЖЕНЫ, ПРЕПОРУЧАЯ ЕЕ КАМЫШОВУ: «Я НЕ ХОЧУ БЫТЬ ПАЛАЧОМ. Я ЖЕНИЛСЯ НА НЕЙ, КОГДА ОНА НИКОГО НЕ ЗНАЛА! Я ЗАСТРЕЛЮСЬ, ЖИВИТЕ ТОЛЬКО СЧАСТЛИВО ${ }^{10}$ !» ПОДОБНОЕ РАЗРЕШЕНИЕ КОНФЛИКТА ЯВНО НАПОМИНАЛО ТРАГИЧЕСКИЙ ФИНАЛ ЖОРЖСАНДОВСКОГО «ЖАКА». НО ПАНАЕВА ЗАСТАВИЛА СВОЮ ГЕРОИНЮ ПРЕВЗОЙТИ В БЛАГОРОДСТВЕ ОБОИХ МУЖЧИН. УСЛЫШАВ СЛОВА УМИРАЮЩЕГО МУЖА, ВОСХИТИВШИСЬ ЕГО «САМООТВЕРЖЕНИЕМ», ОНА ОСТАЛАСЬ С НИМ. «ЛЮБИТЬ ЕГО КАК МУЖА НЕ В МОЕЙ ВЛАСТИ, И ОН САМ ЭТО ХОРОШО ЗНАЕТ; НО ПОКА ОН ЖИВ, Я БУДУ ЕМУ ВЕРНА», - ЗАЯВИЛА ОНА ${ }^{11}$.

СДЕРЖАВ ДАННОЕ СЛОВО, БЕЛКА ЧАХЛА И ХУДЕЛА, СОХРАНЕНИЕ «СВЯТОСТИ» БРАКА НИКОГО ИЗ ТРЕХ УЧАСТНИКОВ ДРАМЫ НЕ СДЕЛАЛО СЧАСТЛИВЫМ. В ОТЛИЧИЕ ОТ ЖОРЖ САНД, КОТОРАЯ, НЕСМОТРЯ НА ЭПИСТОЛЯРНУЮ ФОРМУ РОМАНА, КАК БЫ «УРАВНОВЕШИВАЮЩУЮ» ГЛАВНЫХ ПЕРСОНАЖЕЙ, ВСЕ ЖЕ ПРЕВРАТИЛА ЖАКА В ЦЕНТРАЛЬНУЮ ФИГУРУ (НАСТОЛЬКО ГРАНДИОЗНЫМ БЫЛО ЕГО ВЕЛИКОДУШИЕ, ТАК ПОТРЯСАЛО ПРЕОДОЛЕНИЕ В НЕМ ВСЕГО ЭГОИСТИЧЕСКОГО ВО ИМЯ НОВОЙ ЭТИКИ БРАКА), ПАНАЕВА СДЕЛАЛА ЦЕНТРОМ ЖЕНСКИЙ ХАРАКТЕР. НЕ СЛИШКОМ ГЛУБОКО, НО ТОЧНО ОНА ОЧЕРТИЛА ПСИХОЛОГИЧЕСКОЕ СОСТОЯНИЕ ГЕРОИНИ, В НЕУДОВЛЕТВОРЕННОСТИ ЖИЗНЬЮ КОТОРОЙ МНОГОЕ ШЛО НЕ ТОЛЬКО ОТ «ЖАЖДЫ ЛЮБВИ», НО И ОТ ПРАЗДНОСТИ. БЕЛКА ПРЕДСТАВЛЯЛА СОБОЙ НОВЫЙ ДЛЯ РУССКОЙ ЛИТЕРАТУРЫ ДЕМОКРАТИЧЕСКИЙ ТИП ЖЕНСКОЙ ЛИЧНОСТИ. САМОГО НИЗКОГО (ИЛИ НЕ ВПОЛНЕ ЯСНОГО) ПРОИСХОЖДЕНИЯ, ОНА «ПРИВЫКЛА С ДЕТСТВА К ТРУДУ И СОЗНАНИЮ О ПОЛЬЗЕ, КАКУЮ ОНА ПРИНОСИТ ДРУГИМ ${ }^{12} »$. ПОЯВЛЕНИЕ В ЕЕ ЖИЗНИ ЕЩЕ ОДНОГО МУЖЧИНЫ, ВОПЛОЩАЮЩЕГО ХАРАКТЕР ДИКИЙ, НЕОБУЗДАННЫЙ, ОТРАЖЕННЫЙ В ЕГО ФАМИЛИИ - АТАВИН, - ЕЩЕ БОЛЕЕ ОсЛОЖНИЛО СИТУАЦИЮ. В ОТЛИЧИЕ ОТ СЮЖЕТА РОМАНА Jacques, В КОТОРОМ ПЕРЕМЕШИВАЛИСЬ ЛЮБОВНЫЕ «ТРЕУГОЛЬНИКИ» ЗАПАДНОГО И ВОСТОЧНОГО ТИПА, СТРУКТУРА «ПАСЕКИ» БЫЛА ОСНОВАНА НА ПЕРИПЕТИЯХ ВЫБОРА ЖЕНЩИНОЙ ИЗ НЕСКОЛЬКИХ МУЖЧИН (БЕЛКА ЗЕНОВСКИЙ - КАМЫШОВ - АТАВИН). НО ЛИНИЯ ГЛАВНОЙ ГЕРОИНИ НЕ ИСЧЕРПЫВАЛАСЬ ТОЛЬКО ПРОБЛЕМОЙ ПОИСКОВ ЛЮБВИ. НАПРОТИВ, БЕЛКА ОКАЗАЛАСЬ СПОСОБНОЙ НА СДЕРЖАННОСТЬ, КАК ИСТИННО СЕВЕРНАЯ СЛАВЯНСКАЯ ЖЕНЩИНА. ОНА НЕ ОТДАЛАСЬ СТРАСТИ, ПОДОБНО САНДОВСКОЙ ФЕРНАНДЕ, НО ВНУТРЕННЯЯ БОРЬБА ИСТОЩИЛА ЕЕ СИЛЫ. СТАНОВИЛОСЬ ЯСНО, ЧТО ПЕРЕД НЕЙ ВОЗНИК ВОПРОС О СМЫСЛЕ ДАЛЬНЕЙШЕЙ ЖИЗНИ. ФИНАЛ ПОВЕСТИ ОСТАВАЛСЯ ОТКРЫТЫМ: БЕЛКА ВНОВЬ ВОЗВРАЩАЛАСЬ НА ПАСЕКУ, ГДЕ ШЕСТЬ ЛЕТ НАЗАД ЖИЛА БЕЗЗАБОТНО И СЧАСТЛИВО. НО ТЕПЕРЬ ОНА БЛЕДНА И БОЛЕЗНЕННА. НЕСМОТРЯ НА СВОЕ УВАЖЕНИЕ К МУЖУ, ОНА УШЛА ОТ НЕГО, «ПОТОМУ ЧТО НЕ ЛЮБИЛА ЕГО, И, МОЖЕТ БЫТЬ, ЛЮБИЛА ДРУГОГО, НЕ МОГЛА ПРИТВОРЯТЬСЯ И ОБМАНЫВАТЬ... И ЖИТЬ С НИМ БЫЛО ЕЙ НЕВЫНОСИМО И НЕВОЗМОЖНО ${ }^{13} »$. ЗЕНОВСКИЙ, ПОДОБНО ЖАКУ, ПОКОНЧИЛ С СОБОЙ, О ЧЕМ СООБЩАЛОСЬ ЛАКОНИЧНО И БЕЗ КОММЕНТАРИЕВ: «В ТУ НОЧЬ В ЛЕСУ РАЗДАЛСЯ ВЫСТРЕЛ... ${ }^{14} »$ НО ПРИЧИНОЙ ЕГО САМОУБИЙСТВА БЫЛО ОТЧАЯНИЕ, ТОГДА КАК ЖОРЖСАНДОВСКИЙ ЖАК «САМОУСТРАНИЛСЯ» ВСЛЕДСТВИЕ ПРОДУМАННОГО, ОСОЗНАННО ПРИНЯТОГО РЕШЕНИЯ, И В ЕГО ПОСТУПКЕ СОДЕРЖАЛСЯ ПРОТЕСТ ПРОТИВ МОРАЛИ ЦЕРКОВНОГО БРАКА. ЕСЛИ В РОМАНЕ САНД ДИСКУССИЯ ВОЗНИКАЛА ВОКРУГ ПОСТУПКА МУЖЧИНЫ, ТО У ПАНАЕВОЙ ДИСКУССИОННОСТЬ ПРИОБРЕТАЛА ДАЛЬНЕЙШАЯ СУДЬБА ЖЕНЩИНЫ. НЕ СЛУЧАЙНО ПОСЛЕ СООБЩЕНИЯ О САМОУБИЙСТВЕ ЗЕНОВСКОГО СЛЕДОВАЛА ПРИПИСКА, СНИМАЮЩАЯ ДРАМАТИЗМ ЭТОГО СОБЫТИЯ. «НЕ ЗДЕСЬ ЕЩЕ, - СООБЩАЛ РАССКАЗЧИК, - КОНЕЦ 
ПРИКЛЮЧЕНИЯМ БЕЛКИ. ВТОРАЯ ПОЛОВИНА ЕЕ ЖИЗНИ СОСТАВЛЯЕТ ДРУГУЮ ПОВЕСТЬ, КОТОРУЮ Я, МОЖЕТ БЫТЬ, КОГДА-НИБУДЬ РАССКАЖУ ${ }^{15}$.»

РАСКРЫВАЮТ ЕЕ КОНЦЕПЦИЮ ЛЮБВИ, КОТОРАЯ ВКЛЮЧАЕТ ЭЛЕМЕНТЫ КАК РЕАЛИСТИЧЕСКОЙ, ТАК И ИДЕАЛИСТИЧЕСКОЙ ПАРАДИГМЫ. ПРИРОДА ЛЮБВИ, СОЧЕТАЮЩАЯ ДУХОВНОЕ И ТЕЛЕСНОЕ НАЧАЛО, ОБУСЛОВЛИВАЕТ СЛОЖНОСТЬ ОБРЕТЕНИЯ ГАРМОНИИ. КРИЗИС ИНСТИТУТА СЕМЬИ И БРАКА В СОВРЕМЕННОЙ А. ПАНАЕВОЙ ДЕЙСТВИТЕЛЬНОСТИ ОБУСЛОВИЛ СОСРЕДОТОЧЕННОСТЬ ЕЕ ВНИМАНИЯ НА ОПИСАНИИ НЕДОЛЖНЫХ СИТУАЦИЙ В СФЕРЕ ЛЮБОВНОГО И СЕМЕЙНОГО БЫТА. СИТУАЦИЯ БРАКА ПО РАСЧЕТУ, КОТОРЫЙ ЗАКЛЮЧАЕТСЯ НЕ ТОЛЬКО ПО ИНИЦИАТИВЕ РОДИТЕЛЕЙ, НО И С СОГЛАСИЯ САМИХ ДЕТЕЙ, МЕЧТАЮЩИХ ЛЮБЫМИ СПОСОБАМИ ОСВОБОДИТЬСЯ ОТ РОДИТЕЛЬСКОГО ДЕСПОТИЗМА, РАССМАТРИВАЕТСЯ А. ПАНАЕВОЙ КАК ВАРИАНТ МАТЕРИАЛИЗАЦИИ ЛЮБВИ. В ЛЮБОВНЫХ ОТНОШЕНИЯХ, ОСНОВАННЫХ НА МАТЕРИАЛЬНОЙ ВЫГОДЕ, В УЩЕРБНОМ ПОЛОЖЕНИИ ОКАЗЫВАЕТСЯ ПРЕЖДЕ ВСЕГО ЖЕНЩИНА, КОТОРАЯ НАЧИНАЕТ ОСОЗНАВАТЬ СЕБЯ «ВЕЩНОЙ ПРИНАДЛЕЖНОСТЬЮ», «РАБОЙ» МУЖЧИНЫ («БЕЗОБРАЗНЫЙ МУЖ», «ЖЕНА ЧАСОВОГО МАСТЕРА»).

В ТАКИХ ПРОИЗВЕДЕНИЯХ, КАК «СТЕПНАЯ БАРЫШНЯ» (1855), «МЕРТВОЕ ОЗЕРО» (1851), «ЖЕНСКАЯ ДОЛЯ» (1862) ПАНАЕВА ПОПЫТАЛАСЬ ПРЕДСТАВИТЬ ВАРИАНТЫ МОДЕЛИРОВАНИЯ ГАРМОНИЧНЫХ ВЗАИМООТНОШЕНИЙ МУЖЧИНЫ И ЖЕНЩИНЫ В ЛЮБВИ И БРАКЕ. СИМПТОМАТИЧНО, ЧТО ИДЕАЛЬНЫЕ ЛЮБОВНО-СЕМЕЙНЫЕ ОТНОШЕНИЯ ПЕРСОНАЖЕЙ МОГУТ РАЗВИВАТЬСЯ ТОЛЬКО В ПРОСТРАНСТВЕ ПРИРОДНОГО БЫТИЯ, КОТОРОЕ ОБЕСПЕЧИВАЕТ ГАРМОНИЮ ЛИЧНОСТИ В ЕЕ ОТНОШЕНИЯХ С ЛЮДЬМИ И МИРОМ. ТАК, В ФИНАЛЕ РОМАНА «МЕРТВОЕ ОЗЕРО» ИЗОБРАЖАЕТСЯ ВОССОЗДАНИЕ ЦЕЛОСТНОСТИ СЕМЬИ, ДОМА (МОЛОДЫЕ ГЕРОИ ВМЕСТЕ С РОДИТЕЛЯМИ ОСНОВЫВАЮТ РОДОВОЕ ГНЕЗДО). А В ПОВЕСТИ «СТЕПНАЯ БАРЫШНЯ» ПОЯВЛЯЕТСЯ ОБРАЗ ОЧАРОВАТЕЛЬНОЙ ПРОВИНЦИАЛЬНОЙ ДЕВУШКИ, ЕСТЕСТВЕННОЙ ВО ВСЕХ СВОИХ ПРОЯВЛЕНИЯХ И ЧУЖДОЙ ОБЩЕПРИНЯТЫМ УЛОВКАМ КОКЕТСТВА.

ПРОИЗВЕДЕНИЯ ПАНАЕВОЙ НЕ ТОЛЬКО РЕПРЕЗЕНТИРУЮТ НОВАТОРСКИЕ ЖЕНСКИЕ ХАРАКТЕРЫ, СВЯЗАННЫЕ С УТВЕРЖДЕНИЕМ НОВОЙ ЭТИКИ ЛЮБВИ И БРАКА, НО ВО МНОГОМ ОТРАЖАЮТ ЕЕ СОБСТВЕННЫЕ ПЕРЕЖИВАНИЯ, КОМПЕНСИРУЯ ПРИ ЭТОМ НЕДОСТАТОК ИСПОВЕДАЛЬНЫХ МАТЕРИАЛОВ. О ТОМ, КАК ЭТА ЖЕНЩИНА НАСТРАДАЛАСЬ В «НЕЛЕГАЛЬНОЙ» СИТУАЦИИ, МОЖНО ПОЧУВСТВОВАТЬ ИЗ ЛЮБОВНО-СЕМЕЙНЫХ КОЛЛИЗИЙ, КОТОРЫЕ ОНА ПОСТОЯННО ВАРЬИРОВАЛА В СВОИХ ПОВЕСТЯХ И РОМАНАХ. СИМПАТИИ ПАНАЕВОЙ ВСЕГДА БЫЛИ ОТДАНЫ ГЕРОИНЯМ, ПОСТРАДАВШИМ ОТ ЭГОИЗМА МУЖЧИН И ОТВЕРГНУТЫМ ОБЩЕСТВОМ. В РОМАНЕ «ЖЕНСКАЯ ДОЛЯ», НАПИСАННОМ В ЗНАМЕНАТЕЛЬНЫЙ ДЛЯ НЕЕ ГОД ВСТУПЛЕНИЯ В БРАК С ГОЛОВАЧЕВЫМ (1862), ОНА ВЫРАЗИЛА СВОИ РАЗДУМЬЯ О ЖЕНСКОЙ ЭМАНСИПАЦИИ В ЛИРИКО-ПУБЛИЦИСТИЧЕСКОМ 
ОТСТУПЛЕНИИ. ОБРАЩАЯСЬ К «БЕДНЫМ, ЧЕСТНЫМ ЖЕНЩИНАМ», ПАНАЕВА ПРЕДОСТЕРЕГАЛА ИХ ОТ ИЗЛИШНЕЙ ДОВЕРЧИВОСТИ:

И РАЗВЕ ВЫ НЕ ВИДИТЕ, ЧТО ЖЕНЩИНА, УВЛЕКШАЯСЯ ЭМАНСИПАЦИЕЮ И ОТДАВШАЯСЯ МУЖЧИНЕ БЕЗ ВСЯКИХ ГРАЖДАНСКИХ УСЛОВИЙ, - РАЗВЕ ОНА НЕ ГИБНЕТ ТАКЖЕ В УНИЗИТЕЛЬНОМ РАБСТВЕ И В ПРИДАЧУ ЕЩЕ ОПОЗОРЕННАЯ! ПОВЕРЬТЕ, ПРИ РАЗВРАТЕ ОБЩЕСТВА ВАМ ДАЮТ НАСТОЛЬКО СВОБОДЫ, ЧТОБ БЕЗ ВСЯКИХ ЖЕРТВ СО СТОРОНЫ МУЖЧИН, ВЫ СЛУЖИЛИ БЫ МИНУТНЫМ ПРИХОТЯМ, А ПОТОМ - ТАКЖЕ БЕЗ ВСЯКИХ ЖЕРТВ - ЛЕГКО БЫЛО БЫ И РАЗВЯЗАТЬСЯ С ВАМИ. <... ПОКА САМИ МУЖЧИНЫ НЕ СДЕЛАЮТСЯ НРАВСТВЕННЕЕ - НИКАКАЯ ЭМАНСИПАЦИЯ ЖЕНЩИН НЕВОЗМОЖНА. - РАБЫ НЕ МОГУТ СДЕЛАТЬ СВОБОДНЫМИ РАБЫНЬ! А ЕСЛИ НАСТАНЕТ ИМЕННО НРАВСТВЕННЫЙ ПРОГРЕСС В ЧЕЛОВЕЧЕСТВЕ, ТО ЖЕНЩИНА, БЕЗ ВСЯКИХ ТОЛКОВ И СПОРОВ, ЗАЙМЕТ РАВНОЕ ПОЛОЖЕНИЕ С МУЖчИНОЙ ${ }^{16}$.

В ЦЕЛОМ ПОЛОЖЕНИЕ АВДОТЬИ ЯКОВЛЕВНЫ В РЕДАКЦИИ «СОВРЕМЕННИКА» МОЖНО ОБОЗНАЧИТЬ КАК «ДВОЙСТВЕННОЕ». СОДНОЙ СТОРОНЫ, ОНА ПОЛЬЗОВАЛАСЬ ВНИМАНИЕМ И ПОЧТЕНИЕМ КАК КРАСИВАЯ ЖЕНЩИНА, БЛЕСТЯЩАЯ ХОЗЯЙКА РЕДАКЦИОННЫХ ОБЕДОВ. НО ЕЕ РОЛЬ В РЕДАКЦИИ СВОДИЛАСЬ СОБСТВЕННО К ХОЗЯЙСТВЕННЫМ ДЕЛАМ ДАЖЕ ПОСЛЕ ТОГО, КАК ОНА ВЫСТУПИЛА В КАЧЕСТВЕ ЛИТЕРАТОРА. В ОБЩИХ БЕСЕДАХ ПАНАЕВА ОБЫЧНО НЕ УЧАСТВОВАЛА, ЗАНИМАЯ ПОЗИЦИЮ НЕМОГО НАБЛЮДАТЕЛЯ, СЛУШАТЕЛЯ. ДАЖЕ В КРУГУ «ЛЮДЕЙ СОРОКОВЫХ ГОДОВ» ДЛЯ ТОГО, ЧТОБЫ ПРИНЯТЬ УЧАСТИЕ В МУЖСКИХ ДИСПУТАХ, ВЫСКАЗАТЬ СОБСТВЕННУЮ ТОЧКУ ЗРЕНИЯ, ЕЙ ПРИХОДИЛОСЬ ПРЕОДОЛЕВАТЬ РАСПРОСТРАНЕННЫЕ В ЕЕ МУЖСКОМ ОКРУЖЕНИИ СТЕРЕОТИПНЫЕ ПРЕДСТАВЛЕНИЯ О ТОМ, ЧТО ЖЕНСКАЯ ЛОГИКА НЕ МОЖЕТ ПРЕТЕНДОВАТЬ НА ОБЪЕКТИВНОСТЬ, ЗНАЧИМОСТЬ. ТАК, НА СТРАНИЦАХ СВОИХ «ВОСПОМИНАНИЙ» ОНА ЗАПЕЧАТЛЕЛА ЭПИЗОД ОДНОГО ИЗ ОБЕДЕННЫХ РАЗГОВОРОВ В РЕДАКЦИИ, ГДЕ РЕЧЬ ЗАШЛА О ПОЯВЛЕНИИ В ЛИТЕРАТУРЕ «СЕМИНАРИСТОВ», ТО ЕСТЬ НИКОЛАЯ ДОБРОЛЮБОВА И НИКОЛАЯ ЧЕРНЫШЕВСКОГО. НЕПРИЯЗНЕННОЕ ОТНОШЕНИЕ, ВЫРАЖАЕМОЕ ИВАНОМ ТУРГЕНЕВЫМ И ПАВЛОМ АННЕНКОВЫМ В АДРЕС ЛЮДЕЙ ДРУГОЙ КУЛЬТУРЫ, ИДЕОЛОГИИ И СОЦИАЛЬНОГО СТАТУСА, ПОБУДИЛО ПАНАЕВУ ВЫСКАЗАТЬСЯ В ИХ ЗАЩИТУ. НО УЖЕ САМ ФАКТ ЕЕ ВСТУПЛЕНИЯ В СПОР ВЫЗВАЛ «СМЕХ» И «ТОНКИЕ КОЛКОСТИ» МУЖЧИН, НА ЧТО ОНА ИРОНИЧНО ЗАМЕТИЛА: «ИМЕЕТЕ ПОЛНОЕ ПРАВО СМЕЯТЬСЯ НАДО МНОЙ, ГОСПОДА, ПОТОМУ ЧТО Я САМА НАХОЖУ СМЕШНЫМ, ЧТО ВЗДУМАЛА ВЫСКАЗАТЬ СВОЕ МНЕНИЕ.» (ПАНАЕВА, 1986: 253)

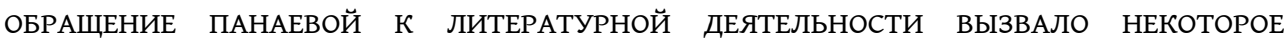
ЗАМЕШАТЕЛЬСТВО В КРУГАХ РЕДАКЦИИ “СОВРЕМЕННИКА», ЧТО МОЖНО ОБЪЯСНИТЬ ДВУМЯ ПРИЧИНАМИ. ЭТО ОБЪЯСНЯЛОСЬ ПОНАЧАЛУ ТЕМ, ЧТО МНОГИЕ НЕ ЗНАЛИ, КТО СКРЫВАЕТСЯ ПОД ПСЕВДОНИМОМ «Н. СТАНИЦКИЙ». ВМЕСТЕ С ТЕМ ПОСЛЕ СМЕРТИ БЕЛИНСКОГО НАМЕТИЛСЯ РАСКОЛ В РЕДАКЦИИ ЖУРНАЛА, МНОГИЕ ЧЛЕНЫ КОТОРОЙ ОТКАЗАЛИСЬ ОТ ИДЕАЛИСТИЧЕСКИХ УСТРЕМЛЕНИЙ СЕРЕДИНЫ 1840-ХГГ. А В ОБЩЕСТВЕННОМ СОЗНАНИИ РОССИИ ВПЛОТЬ ДО НАЧАЛА ХХ В. ФУНКЦИОНИРОВАЛ МИФ О БИОЛОГИЧЕСКОЙ НЕСПОСОБНОСТИ ЖЕНЩИНЫ К ЛЮБЫМ ВИДАМ ИНТЕЛЛЕКТУАЛЬНОЙ ДЕЯТЕЛЬНОСТИ, В ТОМ ЧИСЛЕ ПОЭТИЧЕСКОЙ. С ПОЗИЦИИ ПАТРИАРХАТНОЙ ЛОГИКИ СЧИТАЛОСЬ, ЧТО РЕАЛИЗАЦИЯ ТВОРЧЕСКОЙ ИНДИВИДУАЛЬНОСТИ ЖЕНСКОЙ ЛИЧНОСТИ ДОЛЖНА ОСУЩЕСТВЛЯТЬСЯ В РАМКАХ СЕМЕЙНОГО, ДОМАШНЕГО КРУГА, ПОСКОЛЬКУ ЭТО ОПРАВДАНО ОСОБЕННОСТЯМИ ЖЕНСКОГО ЕСТЕСТВА. ЖЕНЩИНЕ, В СИЛУ ЕЕ ПРИРОДНОЙ СКРОМНОСТИ (В ПАТРИАРХАТНОЙ КУЛЬТУРЕ СКРОМНОСТЬ ВОСПРИНИМАЕТСЯ КАК 
ИСКОННО ЖЕНСКОЕ КАЧЕСТВО), ЯКОБЫ ПРОТИВОЕСТЕСТВЕННО ВЫНОСИТЬ СВОЙ ТВОРЧЕСКИЙ ОПЫТ В ПУБЛИЧНОСТЬ, НА «ПОЗОР», ОТРЫВАТЬСЯ ОТ СВОЕЙ ПРИВЯЗАННОСТИ К СЕМЬЕ И МУЖЧИНЕ.

ПРЕТЕНЗИЯ ЖЕНЩИНЫ НА САМОРЕАЛИЗАЦИЮ В ПРОФЕССИОНАЛЬНОЙ ЛИТЕРАТУРНОЙ СФЕРЕ ВЫЗЫВАЛА НЕГАТИВНУЮ РЕАКЦИЮ ДАЖЕ В КРУГАХ ПРОГРЕССИВНО МЫСЛЯЩИХ ЛЮДЕЙ. НА СТРАНИЦАХ СВОИХ МЕМУАРОВ ПАНАЕВА ЗАПЕЧАТЛЕЛА СИТУАЦИЮ, КОГДА ДАЖЕ БЕЛИНСКИЙ ЯВНО ИСПЫТАЛ ЧУВСТВО НЕДОУМЕНИЯ ПО ПОВОДУ ТОГО, ЧТО ПРОИЗВЕДЕНИЕ, ПОЛУЧИВШЕЕ ЕГО ВЫСОКУЮ ОЦЕНКУ, ОКАЗАЛОСЬ ЛИТЕРАТУРНЫМ ОПЫТОМ ЖЕНЩИНЫ. ОБРАЩАЯСЬ К ПАНАЕВОЙ, КРИТИК СКАЗАЛ:

Я СНАЧАЛА НЕ ХОТЕЛ ВЕРИТЬ НЕКРАСОВУ, ЧТО ЭТО ВЫ НАПИСАЛИ «СЕМЕЙСТВО ТАЛЬНИКОВЫХ» <...> ЕСЛИ БЫ НЕКРАСОВ НЕ НАЗВАЛ ВАС <...> УЖ ИЗВИНИТЕ, Я НИ ЗА ЧТО НЕ ПОДУМАЛ БЫ, ЧТО ЭТО ВЫ. <... Т ТАКОЙ У ВАС ВИД: ВЕЧНО В ХЛОПОТАХ О ХОЗЯЙСТВЕ. <...> Я ДУМАЛ, ЧТО ВЫ ТОЛЬКО О НАРЯДАХ ДУМАЕТЕ. (1986: 172-173)

\section{Заключение}

ЛИЧНАЯ И ТВОРЧЕСКАЯ СУДЬБА А. Я. ПАНАЕВОЙ, ТАЛАНТЛИВОЙ ЖЕНЩИНЫ, СТРЕМЯЩЕЙСЯ РЕАЛИЗОВАТЬ СЕБЯ В ПУБЛИЧНОЙ ДЕЯТЕЛЬНОСТИ, ДРАМАТИЧНА. ЛОМКА ТРАДИЦИОННЫХ СТЕРЕОТИПОВ ПОВЕДЕНИЯ ЖЕНЩИНЫ, НАЧАВШАЯСЯ В РОССИИ В 1840Х ГГ., ПРОИСХОДИЛА БОЛЕЗНЕННО И СЛОЖНО. ОТРАЖЕНИЕ ЭТИХ ПРОЦЕССОВ ПАНАЕВА ЗАПЕЧАТЛЕЛА В СВОЕЙ ПРОЗЕ. В ЦЕНТРЕ ЕЕ ХУДОЖЕСТВЕННОГО МИРА - СУДЬБА ЖЕНСКОГО ПЕРСОНАЖА. ПИСАТЕЛЬНИЦА ИССЛЕДУЕТ СУЩЕСТВОВАНИЕ ЖЕНЩИНЫ В МИРЕ, ОРИЕНТИРОВАННОМ НА ПАТРИАРХАТНЫЕ УСТАНОВКИ, УТВЕРЖДАЮЩЕМ МУЖЧИНУ В КАЧЕСТВЕ НОРМЫ, А ЖЕНЩИНУ - КАК ОТКЛОНЕНИЕ ОТ НЕЕ. В ПАТРИАРХАТНОМ МИРОУСТРОЙСТВЕ ГЕНДЕРНАЯ СИСТЕМА АСИММЕТРИЧНА ТАКИМ ОБРАЗОМ, ЧТО МУЖЧИНА И МУЖСКОЕ ОПРЕДЕЛЯЮТСЯ КАК ОБЩЕЧЕЛОВЕЧЕСКОЕ, А ЖЕНЩИНА И ЖЕНСКОЕ - КАК НЕСАМОСТОЯТЕЛЬНОЕ, ВТОРОСТЕПЕННОЕ, ПРИВАТНОЕ.

ПАНАЕВА ОПИСЫВАЕТ ТРИ ВАРИАНТА ЖИЗНЕННОГО СЦЕНАРИЯ ЖЕНЩИНЫ. ПЕРВЫЙ

СВЯЗАН С ПОДЧИНЕНИЕМ, МИМИКРИРОВАНИЕМ ПОД МАСКУЛИННЫЕ ТРЕБОВАНИЯ, ВТОРОЙ - С ПРОТЕСТОМ ПРОТИВ НИХ, ТРЕТИЙ - С СОЗИДАНИЕМ ГАРМОНИЧНОГО ПРОСТРАНСТВА БЫТИЯ. ПИСАТЕЛЬНИЦА НАИБОЛЕЕ ПОЛНО ПРЕДСТАВИЛА ПЕРВЫЕ ДВЕ МОДЕЛИ КАК НАИБОЛЕЕ РАСПРОСТРАНЕННЫЕ В РЕАЛЬНОЙ ДЕЙСТВИТЕЛЬНОСТИ, ТРЕТИЙ - ТОЛЬКО НАМЕЧЕН В ЕЕ ПРОИЗВЕДЕНИЯХ.

ГЕРОИНИ, ИЗБРАВШИЕ ПУТЬ ПРИСПОСОБЛЕНИЯ К ЛЕГИТИМНЫМ В ПАТРИАРХАТНОМ СОЦИУМЕ НОРМАМ ЖЕНСКОГО БЫТИЯ, ИМЕЮТ ВОЗМОЖНОСТЬ СУЩЕСТВОВАТЬ ТОЛЬКО В РОЛЯХ ХОРОШЕЙ ХОЗЯЙКИ, ЛЮБЯЩЕЙ И ПРЕДАННОЙ ЖЕНЫ, ДОБРОЙ МАТЕРИ. ПАНАЕВА ОБНАРУЖИВАЕТ ТТРАГЕДИЮ НЕВОПЛОЩЕННОСТИ» ЖЕНСКОЙ ЛИЧНОСТИ, КОТОРАЯ НЕ МОЖЕТ ОБРЕСТИ ГАРМОНИЮ В ОКРУЖАЮЩЕМ МИРЕ. РЕАЛЬНЫЕ ОБСТОЯТЕЛЬСТВА, А ТАКЖЕ ГЕНДЕРНЫЕ СТЕРЕОТИПЫ, РЕГУЛИРУЮЩИЕ ПОВЕДЕНИЕ ЧЕЛОВЕКА В ЗАВИСИМОСТИ ОТ ЕГО БИОЛОГИЧЕСКОГО ПОЛА, ОБУСЛОВЛИВАЮТ НРАВСТВЕННОПСИХОЛОГИЧЕСКУЮ И СОЦИАЛЬНУЮ РАСТЕРЯННОСТЬ И НЕУВЕРЕННОСТЬ ЖЕНЩИНЫ.

ВМЕСТЕ С ТЕМ АВТОР ЖЕНЩИНА ПОКАЗЫВАЕТ, ЧТО, НЕСМОТРЯ НА ТОТАЛЬНУЮ ПОДЧИНЕННОСТЬ, ЗАВИСИМОСТЬ ГЕРОИНЬ В СЕМЬЕ И ОБЩЕСТВЕ, У НИХ ВСЕГДА СОХРАНЯЕТСЯ ВОЗМОЖНОСТЬ ВЫБОРА ИНОЙ ЖИЗНЕННОЙ ПОЗИЦИИ. ЛУЧШИЕ ГЕРОИНИ ПАНАЕВОЙ ЧЕРЕЗ ВЫПАВШИЕ НА ИХ ДОЛЮ ИСПЫТАНИЯ ВЫХОДЯТ К ОБРЕТЕНИЮ СМЫСЛА 
СОБСТВЕННОГО СУЩЕСТВОВАНИЯ. ПРИ ЭТОМ НЕГАТИВНЫЕ СЦЕНАРИИ ЖЕНСКОГО СУЩЕСТВОВАНИЯ (ПОДЧИНЕНИЕ, ЯВНЫЙ ИЛИ СКРЫТЫЙ ПРОТЕСТ), КАК ПРАВИЛО, РЕАЛИЗУЮТСЯ В ПРОСТРАНСТВЕ ГОРОДСКОЙ ЦИВИЛИЗАЦИИ. ПРЕБЫВАНИЕ ЖЕНЩИНЫ В ПЕТЕРБУРГСКОЙ СРЕДЕ, ВОПЛОЩАЮЩЕЙ ВСЕ АТРИБУТЫ ЦИВИЛИЗОВАННОГО МИРА, ХАРАКТЕРИЗУЕТСЯ ПРЕДЕЛЬНОЙ НЕСВОБОДОЙ.

ВОЗМОЖНОСТЬ ПОЛНОЦЕННОГО СУЩЕСТВОВАНИЯ ЖЕНЩИНЫ ПАНАЕВА СВЯЗЫВАЕТ С ПРИРОДНЫМ, УСАДЕБНЫМ МИРОМ. ИМЕННО В ПРОСТРАНСТВЕ УСАДЕБНОЙ КУЛЬТУРЫ, ПРЕДПОЛАГАЮЩЕЙ СИНТЕЗ ЕСТЕСТВЕННОГО И ЦИВИЛИЗОВАННОГО НАЧАЛ, ГЕРОИНЯ СПОСОБНА СОЗДАТЬ ГАРМОНИЧНЫЙ МИР И МАКСИМАЛЬНО РЕАЛИЗОВАТЬ СВОЙ ЛИЧНОСТНЫЙ ПОТЕНЦИАЛ (ПОВЕСТИ «ПАСЕКА», «СТЕПНАЯ БАРЫШНЯ») ${ }^{18}$.

ТАКИМ ОБРАЗОМ, ЛИЧНАЯ И ТВОРЧЕСКАЯ СУДЬБА АВДОТЬИ ПАНАЕВОЙ ОТРАЖАЕТ ДРАМАТИЗМ ПОЛОЖЕНИЯ НЕЗАУРЯДНОЙ РУССКОЙ ЖЕНЩИНЫ, РЕШИВШЕЙСЯ ПРОТИВОСТОЯТЬ ПАТРИАРХАТНОЙ ТРАДИЦИИ КАК СВОИМ ПОВЕДЕНИЕМ, ТАК И ПУБЛИЧНОЙ ТВОРЧЕСКОЙ ДЕЯТЕЛЬНОСТЬЮ.

\section{BIBLIOGRAPHIE}

БРАЗОЛЕНКО Б. (1908), ЛЕКЦИИ ПО ИСТОРИИ И ЛИТЕРАТУРЕ. РУССКАЯ ЖЕНЩИНА В ЖИЗНИ И ЛИТЕРАТУРЕ, САНКТ-ПЕТЕРБУРГ: ИЗДАТЕЛЬСТВО ВЕСТНИКА ЗНАНИЯ В. В. БИТНЕРА.

КАФАНОВА ОЛЬГА БОДОВНА (1998а), ЖОРЖ САНД И РУССКАЯ ЛИТЕРАТУРА ХІХ ВЕКА (МИФЫ И РЕАЛЬНОСТЬ). 1830-1860-Е ГГ, ТОМСК: ИЗДАТ. ЛАБОРАТОРИЯ ТГПУ.

КАФАНОВА ОЛЬГА БОДОВНА (1998b), «ЛЮБОВНЫЙ БЫТ “ЛЮДЕЙ СОРОКОВЫХ ГОДОВ”», ВЕСТНИК ТОМСКОГО ГОС. УН-ТА. НАУЧНЫЙ ЖУРНАЛ (ГУМАНИТАРНЫЙ СПЕЦ. ВЫПУСК ИСТОРИЧЕСКОГО, ФИЛОЛОГИЧЕСКОГО И КУЛЬТУРОЛОГИЧЕСКОГО ФАКУЛЬТЕТОВ), 266, 78-87.

ПАНАЕВА АВДОТЬЯ (ГОЛОВАЧЕВА) (1986), ВОСПОМИНАНИЯ, МОСКВА: ПРАВДА.

ПЫПИН А. Н. (1905), Н. А. НЕКРАСОВ, САНКТ-ПЕТЕРБУРГ: ТИП. М. М. СТАСЮЛЕВИЧА.

СКАТОВ НИКОЛАЙ НИКОЛАЕВИЧ (1994), НЕКРАСОВ, МОСКВА: МОЛОДАЯ ГВАРДИЯ.

СТАНИЦКИЙ Н. (1848), «БЕЗОБРАЗНЫЙ МУЖ. ПОВЕСТЬ В ПИСЬМАХ», СОВРЕМЕННИК, Т. 8, АПРЕЛЬ, отд. І.

ТАТАРКИНА СВЕТЛАНА ВЛАДИМИРОВНА (2006), ТВОРЧЕСТВО А. Я. ПАНАЕВОЙ В ЛИТЕРАТУРНОМ КОНТЕКСТЕ ЭПОХИ: ГЕНДЕРНЫЙ АСПЕКТ, ДИССЕРТАЦИЯ НА СОИСКАНИЕ СТЕПЕНИ КАНД. ФИЛОЛОГИЧЕСКИХ НАУК, ТОМСК.

ХАЙДСБРАНД Р. \& ВИНКО С. (1999), «РАБОТА С ЛИТЕРАТУРНЫМ КАНОНОМ: ПРОБЛЕМА ТЕНДЕРНОЙ ДИФФЕРЕНЦИАЦИИ ПРИ ВОСПРИЯТИИ (РЕЦЕПЦИИ) И ОЦЕНКЕ ЛИТЕРАТУРНОГО ПРОИЗВЕДЕНИЯ», ПОЛ. ТЕНДЕР. КУЛЬТУРА: НЕМЕЦКИЕ И РУССКИЕ ИССЛЕДОВАНИЯ, ПОД РЕД. Э. ШОРЕ И К. ХАЙДСР., М., 21-79.

ЧУКОВСКИЙ КОРНЕЙ И. (1922), ЖЕНА ПОЭТА. (АВДОТЬЯ ЯКОВЛЕВНА ПАНАЕВА), ПЕТРОГРАД: «ЭПОХА». 
ЧУКОВСКИЙ КОРНЕЙ И. (1928), «О СЕМЕЙСТВЕ ТАЛЬНИКОВЫХ», А. Я. ПАНАЕВА, СЕМЕЙСТВО ТАЛЬНИКОВЫХ: ПОВЕСТЬ, ЛЕНИНГРАД: Academia.

ШАШКОВ СЕРАФИМ СЕРАФИМОВИЧ (1879), ИСТОРИЯ РУССКОЙ ЖЕНЩИНЫ, САНКТ-ПЕТЕРБУРГ:

ТИПОГРАФИЯ А. С. СУВОРИНА.

ЩЕРБИНА НИКОЛАЙ ФЕДОРОВИЧ (1929), АЛЬБОМ ИПОХОНДРИКА. ЭПИГРАММЫ И САТИРЫ, ЛЕНИНГРАД: тип. приБой.

\section{NOTES}

1. САМА ПАНАЕВА, ОЧЕНЬ СДЕРЖАННАЯ И ТАКТИЧНАЯ В ОПИСАНИИ СВОЕЙ СУПРУЖЕСКОЙ ЖИЗНИ, ПРИВОДИТ ТЕМ НЕ МЕНЕЕ ФАКТЫ, КРАСНОРЕЧИВО ПОКАЗЫВАЮЩИЕ, ЧТО ЕЕ МУЖ, И. И. ПАНАЕВ НИКОГДА НЕ ОТКАЗЫВАЛСЯ ОТ ХОЛОСТЯЦКИХ ПРИВЫЧЕК. ОН МОГ, НАПРИМЕР, ПОСЛЕ ДРУЖЕСКОГО ОБЕДА В ПАРИЖСКОМ РЕСТОРАНЕ ОСЕНЬЮ 1844 Г. ОТПРАВИТЬСЯ С БОТКИНЫМ И ОГАРЕВЫМ «НА КАКОЙ-ТО БАЛ, ГДЕ ВЕСЕЛЯТСЯ ГРИЗЕТКИ», В ТО ВРЕМЯ КАК М. БАКУНИН ПРОВОЖАЛ ЕГО ЖЕНУ ДОМОЙ (ПАНАЕВА, 1986: 129).

2. «ЗАПИСКИ ВАСИЛИЯ АНТОНОВИЧА ИНСАРСКОГО», РУССКАЯ СТАРИНА, 1895, ЯНВАРЬ, № 1, С. 112.

3. Т. Н. ГРАНОВСКИЙ И ЕГО ПЕРЕПИСКА, МОСКВА: ТОВАРИЩЕСТВО ТИПОГРАФИИ А. И. МАМОНТОВА, 1897, Т. 2, С. 284.

4. ТАМ ЖЕ, С. 288.

5. ТАМ ЖЕ, С. 289.

6. МАРИЯ СЕМЕНОВНА ЖУКОВА (1805-1855) ВПЕРВЫЕ В РУССКОЙ ЛИТЕРАТУРЕ ИСПОЛЬЗОВАЛА СЮЖЕТ «САМОУСТРАНЕНИЯ» МУЖА, ПРЕДЛОЖЕННЫЙ ЖОРЖ САНД В РОМАНЕ «ЖАК». В ПОВЕСТИ «БАРОН РЕЙХМАН» ИЗ ЦИКЛА «ВЕЧЕРА НА КАРПОВКЕ» (1837) ГЕНЕРАЛ РЕЙХМАН, УЗНАВШИЙ О ЛЮБВИ СВОЕЙ ЖЕНЫ К ДРУГОМУ, ВЕДЕТ СЕБЯ СОВЕРШЕННО НЕ ТРАДИЦИОННО, ДАВАЯ ЕЙ СВОБОДУ РАСПОРЯЖАТЬСЯ СОБОЙ. СМ. Olga Kafanova, Les « Sœurs russes » de Georges Sand, ЭЛЕКТР. РЕСУРС: <http://institut-est-ouest.ens-lyon.fr/spip.php?rubrique107\&lang=frjournée d'études>.

7. ПёТР НИКОЛАЕВИЧ КУДРЯВЦЕВ (1816-1858) - ЛИТЕРАТОР И ПРОФЕССОР МОСКОВСКОГО УНИВЕРСИТЕТА, В СВОЕМ ТВОРЧЕСТВЕ БЫЛ ЗАЩИТНИКОМ ЖЕНСКОЙ СВОБОДЫ В ЧУВСТВАХ. В ПОВЕСТИ «БЕЗ РАССВЕТА» (1847), СЮЖЕТНО ПЕРЕКЛИКАЮЩЕЙСЯ С РОМАНОМ Ж. САНД «ИНДИАНА», КУДРЯВЦЕВ ПОКАЗАЛ СТРАДАНИЯ МОЛОДОЙ УМНОЙ ЖЕНЩИНЫ В БРАКЕ БЕЗ ЛЮБВИ С ПОШЛЫМ И РАСПУТНЫМ ЧЕЛОВЕКОМ.

8. СОВРЕМЕННИК, 1849, Т. 13, ФЕВРАЛЬ, ОТД. V, С. 168-181.

9. ТАМ ЖЕ, 1849, Т. 18, НОЯБРЬ, ОТД. І, С. 51.

10. TAM ЖЕ, С. 53.

11. TAM ЖЕ, С. 55.

12. TAM ЖЕ, С. 56.

13. ТАМ ЖЕ, С. 93.

14. TAM ЖЕ, С. 94.

15. ТАМ ЖЕ.

16. ЖЕНСКАЯ ДОЛЯ. РОМАН Н. СТАНИЦКОГО, В: СОВРЕМЕННИК, 1862, Т. 92, МАРТ, С. 50-51.

17. ТЕРМИН «ПАТРИАРХАТ» (БУКВ. ВЛАСТЬ ОТЦА, ТАКЖЕ АНДРАРХИЯ, АНДРОКРАТИЯ) ОБОЗНАЧАЕТ ФОРМУ СОЦИАЛЬНОЙ ОРГАНИЗАЦИИ, В КОТОРОЙ МУЖЧИНА ЯВЛЯЕТСЯ ОСНОВНЫМ НОСИТЕЛЕМ ПОЛИТИЧЕСКОЙ ВЛАСТИ И МОРАЛЬНОГО АВТОРИТЕТА, ОСУЩЕСТВЛЯЕТ КОНТРОЛЬ НАД СОБСТВЕННОСТЬЮ, А ОТЦЫ В СЕМЬЯХ ОБЛАДАЮТ ЛИДИРУЮЩИМ ПОЛОЖЕНИЕМ. ДЛЯ ПАТРИАРХАТА ХАРАКТЕРНО НАЛИЧИЕ ИНСТИТУТОВ МУЖСКОЙ ВЛАСТИ И МУЖСКИХ ПРИВИЛЕГИЙ, А ТАКЖЕ ПОДЧИНЁННОЕ ПОЛОЖЕНИЕ ЖЕНЩИН. В КОНТЕКСТЕ КУЛЬТУРЫ ХІХ В. 
ТЕРМИН «ПАТРИАРХАТ» ПРИОБРЕТАЕТ НЕГАТИВНУЮ КОННОТАЦИЮ, ПОСКОЛЬКУ ПРЕДПОЛАГАЕТ НАМЕРЕННОЕ ПОДАВЛЕНИЕ ЖЕНСКОЙ ЛИЧНОСТИ. «ПАТРИАРХАЛЬНОСТЬ» НЕ ИМЕЕТ ЭТОГО НЕГАТИВНОГО ЗНАЧЕНИЯ, ОБОЗНАЧАЯ ВЕРНОСТЬ ОТЖИВШЕЙ СТАРИНЕ, СТАРОЗАВЕТНОСТЬ.

18. СМ. ТАТАРКИНА (2006).

\section{RÉSUMÉS}

СУДЬБА АВДОТЬИ ПАНАЕВОЙ СВЯЗАНА С ЛОМКОЙ ТРАДИЦИОННЫХ ЭТИЧЕСКИХ СТЕРЕОТИПОВ НАЧАВШЕЙСЯ В РОССИИ В 1840-Х ГГ.-1860-Х ГГ. ДЛЯ РУССКОЙ ЖЕНЩИНЫ БЫЛО ОЧЕНЬ ТРУДНО ВЫЙТИ ИЗ ЛИЧНОГО ПРОСТРАНСТВА И ВОЙТИ В ПРОСТРАНСТВО ПУБЛИЧНОЕ. СИТУАЦИЯ А. ПАНАЕВОЙ ОСЛОЖНИЛАСЬ ЛЮБОВНЫМ ТРЕУГОЛЬНИКОМ, В КОТОРОМ ОНА ОКАЗАЛАСЬ МЕЖДУ СВОИМ ЗАКОННЫМ МУЖЕМ (ИВАНОМ ПАНАЕВЫМ) И МУЖЕМ ГРАЖДАНСКИМ (НИКОЛАЕМ НЕКРАСОВЫМ); ОБА БЫЛИ ДРУЗЬЯМИ И СОРЕДАКТОРАМИ ЗНАМЕНИТОГО ЖУРНАЛА «СОВРЕМЕННИК». ОТРАЖЕНИЕ ЭТИХ СЛОЖНЫХ ПРОЦЕССОВ ПАНАЕВА ЗАПЕЧАТЛЕЛА В СВОЕЙ ПРОЗЕ, КОМПОЗИЦИОННО И СЮЖЕТНО ВЫДВИГАЮЩЕЙ ГЕРОИНЮ-ЖЕНЩИНУ НА ПЕРВЫЙ ПЛАН. В ЦЕНТРЕ ЕЕ ХУДОЖЕСТВЕННОГО МИРА - СУДЬБА ЖЕНСКОГО ПЕРСОНАЖА, СУЩЕСТВУЮЩЕГО В УСЛОВИЯХ ПАТРИАРХАТНЫХ ТРАДИЦИЙ И В РАЗНОЙ СТЕПЕНИ ПРОТИВОСТОЯЩЕГО ИМ. СОБСТВЕННАЯ ЛИЧНАЯ И ТВОРЧЕСКАЯ ЖИЗНЬ ПИСАТЕЛЬНИЦЫ РЕПРЕЗЕНТИРУЕТ ДРАМАТИЗМ ЕЕ ПОПЫТОК СОПРОТИВЛЕНИЯ ТРАДИЦИОННЫМ ПРЕДСТАВЛЕНИЯМ О ПРЕДНАЗНАЧЕНИИ ЖЕНЩИНЫ.

Le destin d'Avdotia Panaéva s'explique par la transformation des stéréotypes éthiques traditionnels qui s'est amorcée en Russie dans les années 1840-1860. Il était très difficile pour une femme russe de sortir de l'espace intime et d'entrer dans l'espace public. La situation d'Avdotia Panaéva était compliquée par le triangle amoureux dans lequel elle se trouvait entre son mari légitime (Ivan Panaev) et son mari «civil» (Nikolaï Nékrassov), tous deux étant amis et rédacteurs de la revue célèbre Le Contemporain. La prose de Panaéva reflète ces processus complexes, en mettant au premier plan, par sa composition et par ses thèmes, l'héroïne-femme. Au centre de sa poétique se trouve le destin du personnage féminin plongé dans les conditions du patriarcat, les adoptant ou, au contraire, s'y opposant dans différentes mesures. La vie privée et publique de l'écrivaine elle-même illustre sa tentative dramatique de résister aux idées traditionnelles sur la destinée de la femme.

\section{INDEX}

Mots-clés : espace privé et public, triangle d'amour, éthique d'amour, prose féministe, personnage féminin, transformation des stéréotypes traditionnels, patriarcat motsclesru ЛИЧНОЕ И ПУБЛИЧНОЕ ПРОСТРАНСТВО, ЛЮБОВНЫЙ ТРЕУГОЛЬНИК, ЛЮБОВНАЯ ЭТИКА, ЖЕНСКАЯ ПРОЗА, ЖЕНСКИЙ ПЕРСОНАЖ, ЛОМКА ТРАДИЦИОННЫХ СТЕРЕОТИПОВ, ПАТРИАРХАТ 


\section{AUTEUR}

\section{ОЛЬГА КАФАНОВА}

Université de la flotte fluviale et de la marine de Saint-Pétersbourg, Université de Tomsk (Russie) 